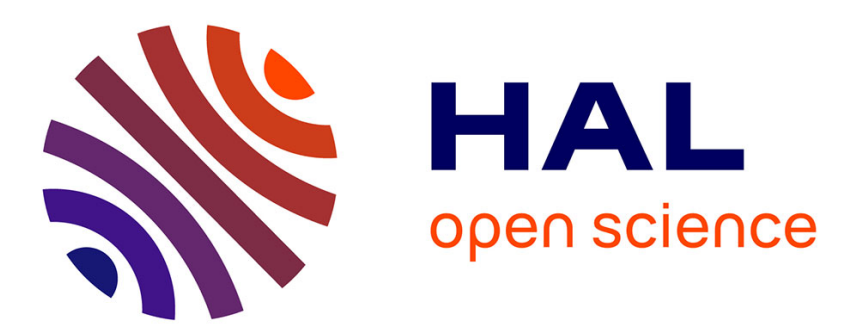

\title{
Essai de caractérisation des matériaux vitreux à partir des données relatives au phénomène de dévitrification thermique
}

\author{
M. Murat, A. Bachiorrini, A. Negro
}

\section{- To cite this version:}

M. Murat, A. Bachiorrini, A. Negro. Essai de caractérisation des matériaux vitreux à partir des données relatives au phénomène de dévitrification thermique. Revue de Physique Appliquée, 1977, 12 (5), pp.653-666. 10.1051/rphysap:01977001205065300 . jpa-00244221

HAL Id: jpa-00244221

https://hal.science/jpa-00244221

Submitted on 1 Jan 1977

HAL is a multi-disciplinary open access archive for the deposit and dissemination of scientific research documents, whether they are published or not. The documents may come from teaching and research institutions in France or abroad, or from public or private research centers.
L'archive ouverte pluridisciplinaire HAL, est destinée au dépôt et à la diffusion de documents scientifiques de niveau recherche, publiés ou non, émanant des établissements d'enseignement et de recherche français ou étrangers, des laboratoires publics ou privés. 


\title{
ESSAI DE CARACTÉRISATION DES MATÉRIAUX VITREUX A PARTIR DES DONNÉES RELATIVES AU PHÉNOMÈNE DE DÉVITRIFICATION THERMIQUE
}

\author{
M. MURAT \\ I. N. S. A. de Lyon, Laboratoire de Chimie Appliquée 404, INSA 20, 69621 Villeurbanne, France
}

A. BACHIORRINI et A. NEGRO

Politecnico de Turin (Italie), Istituto di Chimica Generale e Applicata

\begin{abstract}
Résumé. - La façon dont se manifeste la dévitrification thermique d'un matériau vitreux, phénomène mis en évidence par des techniques expérimentales appropriées (Analyse thermique, radiocristallographie spectrographie d'absorption IR, etc...) peut apporter des informations intéressantes concernant la structure du verre de départ.

L'enregistrement d'une courbe d'ATD met en évidence un ou plusieurs pics de dévitrification. La connaissance, par analyse radiocristallographique, de la nature des phases qui cristallisent lors du traitement thermique, permet d'établir certaines corrélations entre la composition chimique et la stabilité thermique du matériau de départ.

La mise en œuvre de techniques expérimentales annexes (dilatométrie, microscopie électronique à balayage, ...) montre que dans certains cas le phénomène de dévitrification thermique est complexe et peut être associé à un frittage du matériau surtout lorsque celui-ci est initialement à l'état pulvérulent.

On présente un certain nombre d'exemples caractéristiques tirés de recherches sur des verres de silico-aluminate et sur des laitiers vitreux, matériaux qui peuvent être utilisés pour l'élaboration de vitrocéramiques.

On montre en particulier que la présence de certains cations modificateurs de réseau $\left(\mathrm{Mg}^{2+}, \mathrm{Fe}^{3+}\right)$ abaisse la stabilité thermique du verre alors qu'un effet inverse se manifeste lorsque le verre contient des quantités importantes d'alumine.
\end{abstract}

Abstract. - The process by which the thermal devitrification of vitreous materials happens, phenomenon detected by appropriate experimental technics (thermal analysis, X-ray crystallography, IR spectroscopy, ...) can bring interesting data about the structure of original glasses.

The recording of DTA curves exhibits one or several devitrification peaks. The knowledge, by X-ray crystallography, of the nature of the crystallizing phases during heating allows to establish certain correlations between the chemical composition and the thermal stability of the glasses.

The use of particular experimental technics (dilatometry, scanning electron microscopy, ...) shows that in certain cases the devitrification phenomenon is complex and can be associated with a sintering of the material, particularly when it is in a powdered state.

A lot of characteristical examples from authors' researches about silico-alumina glasses and about vitreous blast-furnace or synthetic slags (which can be used as raw-materials in the manufacture of glass-ceramics) are presented.

It is particularly pointed out that certain lattice modyfiers such as $\mathrm{Mg}^{2+}$ and $\mathrm{Fe}^{3+}$ lower the thermal stability of the glasses, whereas an inverse effect is observed when the glasses content of alumina becomes high.

1. Introduction. Généralités. - 1.1 VERRES ET VITROCÉRAMIQUES [1-2]. - Le refroidissement lent d'un liquide conduit généralement à un phénomène de cristallisation (Fig. 1a). Cependant certains liquides $\left(\mathrm{SiO}_{2}, \mathrm{GeO}_{2}, \mathrm{P}_{2} \mathrm{O}_{5}\right.$, glycérine, mélanges d'oxydes, éléments divers, ... tous à l'état fondu) soumis au refroidissement manifestent un phénomène de surfusion puis conservent un état rigide avec existence d'un certain ordre à courte distance. On obtient des verres ou matériaux amorphes dont les propriétés thermodynamiques diffèrent de celles du même matériau à l'état cristallisé (Fig. 1a).
En fait l'aptitude d'une fonte à recristalliser au refroidissement dépend de la position respective des courbes de variation de la vitesse de nucléation et de la vitesse de croissance (Fig. $1 b$ et Fig. 1c). On obtient un verre lorsque ces deux courbes présentent un recouvrement minimum.

Les verres ont tendance à recristalliser lors d'un traitement thermique ultérieur et l'élaboration des vitrocéramiques est basée sur ce phénomène, les propriétés des matériaux obtenus étant fonction de la composition du verre de départ et du protocole de réalisation de la nucléation (formation des germes de la 
$H$ enthalpie molaire

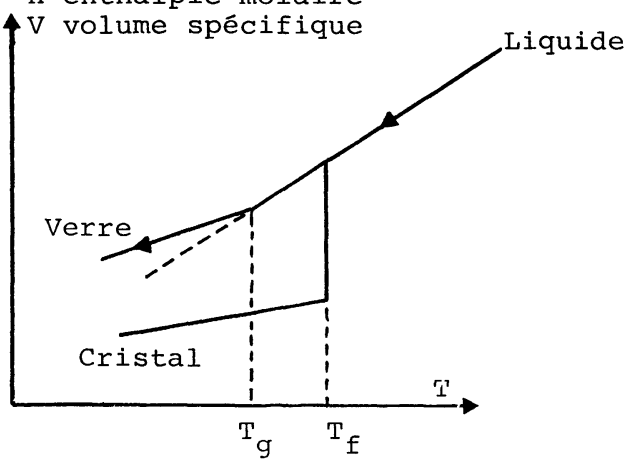

(a)

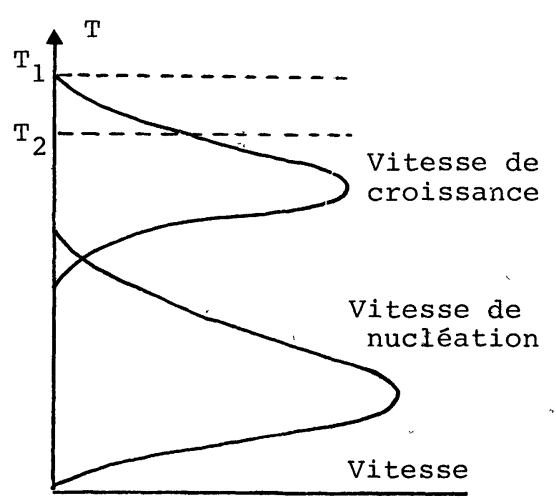

(b)

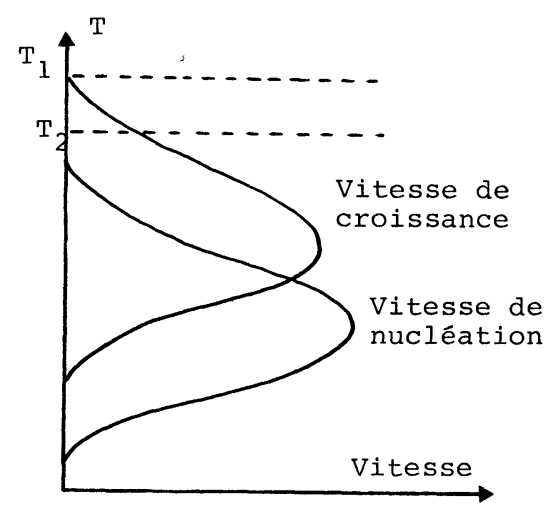

(c)

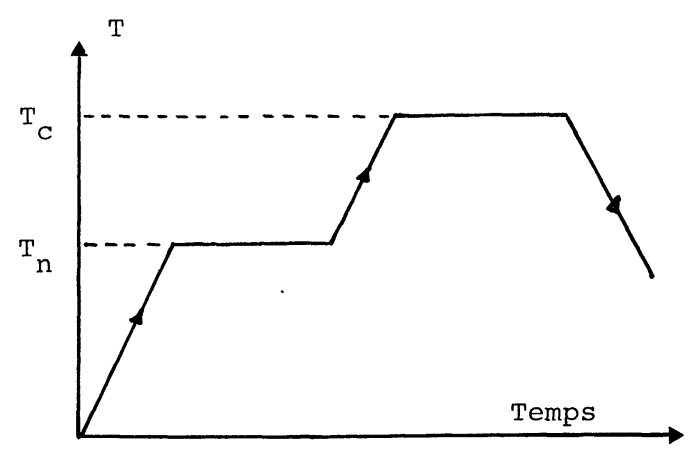

(d)

FIG. 1. - a) Propriétés thermodynamiques du verre et du cristal ; b) et c) Variation de la vitesse de nucléation et de la vitesse de croissance lors de la cristallisation d'un verre ; $d$ ) Cycle thermique pour l'élaboration d'une vitrocéramique. phase cristallisée) et de la croissance de ces germes. Pour obtenir une bonne vitrocéramique on aura intérêt à créer de nombreux germes dont la croissance ultérieure conduira à un matériau microcristallisé. Pour ce faire, le verre de départ est additionné ou non d'agents de nucléation, puis chauffé (vitesse de chauffe de 2 à $5 \% / \mathrm{mn}$ ) jusqu'à une température $T_{\mathrm{N}}$ (Fig. $1 d$ ) correspondant au maximum de la vitesse de nucléation (viscosité de $10^{11}$ à $10^{12}$ poises). La température est maintenue à $T_{\mathrm{N}}$ pendant 2 à $3 \mathrm{~h}$, puis on l'élève à nouveau et suffisamment lentement $(5 \% / \mathrm{mn})$ pour que se produise la croissance de la phase cristallisée, et ceci jusqu'à une température $T_{\mathrm{C}}$ ou température de cristallisation. Enfin le matériau est refroidi lentement.

Afin d'assurer les meilleures conditions d'obtention des vitrocéramiques, il est nécessaire de caractériser la matière première (détermination des températures de ramollissement, de formation des germes, de cristallisation, de première fusion). L'analyse thermique différentielle (ATD) est particulièrement bien adaptée à ce problème, aussi l'avons-nous largement utilisée.

1.2 ANALYSe THERMiQue Des VerRes [3]. - La courbe ATD d'un verre ou d'un matériau vitreux présent un ou plusieurs pics exothermiques précédés généralement d'un pic ou déflexion endothermique de plus faible intensité (Fig. 2). Les températures auxquelles se manifestent ces phénomènes dépendent de la composition et de la structure du verre.

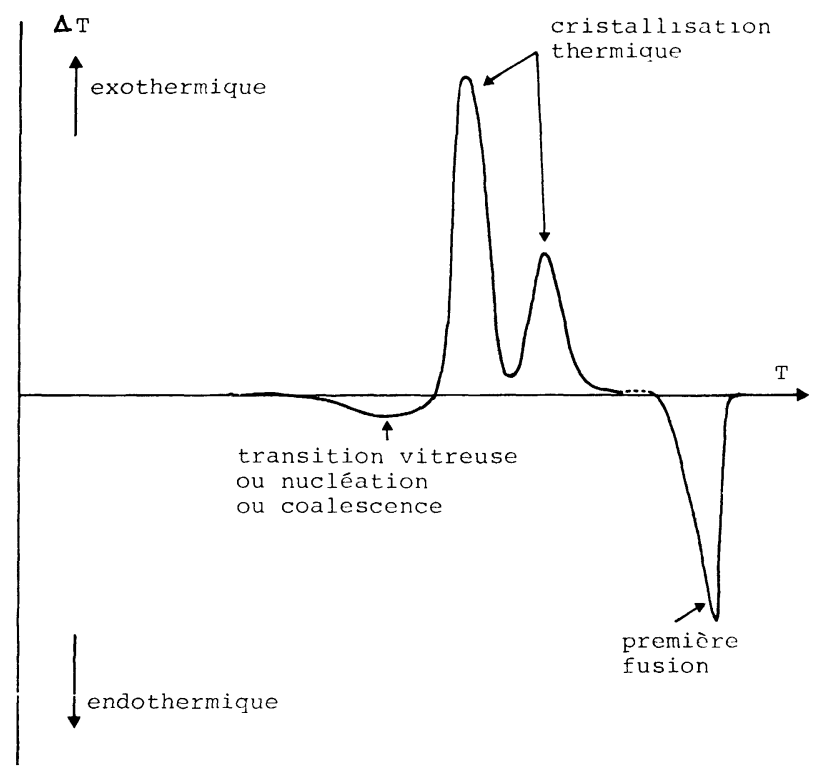

FIG. 2. - Allure type de la courbe ATD d'un verre.

L'effet endothermique se produit dans le domaine de température des transformations vitreuses (variations des propriétés physiques) ou du $T_{\mathrm{g}}$. Il peut être également associé à une coalescence du matériau ou à la nucléation des phases cristallisées.

Le ou les effets exothermiques traduisent la dévitrification du matériau (croissances des germes) et cor- 
respond à un phénomène qui peut être très rapide (pic très aigu) ou lent (pic large). Ceci conduit à penser que la hauteur du pic exothermique est une mesure qualitative de la vitesse de cristallisation.

Nous ne discuterons pas ici en détail d'autres phénomènes qui peuvent se produire après la dévitrification (interactions entre les phases cristallisées, point de transition polymorphiques ou transformation du deuxième ordre de ces phases, point de première fusion).

L'allure des courbes ATD des verres dépend de certaines caractéristiques physiques du matériau, et en particulier de la granulométrie. Les avis concernant l'influence de ce facteur sont assez partagés : Thakur et al. [4] ont montré par exemple que dans le cas des verres du système $\mathrm{Li}_{2} \mathrm{O}-\mathrm{Al}_{2} \mathrm{O}_{3}-\mathrm{SiO}_{2}$ la dimension des grains de verre n'affecte pas sensiblement l'effet endothermique lié à la transformation vitreuse, mais les verres en grains fins présentent une dévitrification à température plus basse que les verres en gros grains. Un phénomène inverse a été observé par Mercier [5] dans le cas des verres du système

$$
\mathrm{Li}_{2} \mathrm{O}-\mathrm{K}_{2} \mathrm{O}-\mathrm{TiO}_{2}-\mathrm{Al}_{2} \mathrm{O}_{3}-\mathrm{SiO}_{2} \text {. }
$$

L'influence de la granulométrie modifie également la forme des pics : ceux-ci deviennent de plus en plus pointus quand la dimension des grains diminue, phénomène qui est variable selon la cristallisation considérée.

La vitesse de chauffe modifie aussi la position en température du ou des pics exothermiques de cristallisation, mais n'a pas d'influence marquée sur l'effet endothermique associé à la transformation vitreuse.

L'enregistrement de la courbe ATD d'une matière première vitreuse à partir de laquelle on veut préparer une vitrocéramique présente donc un certain intérêt car elle permet de définir les domaines de température dans lesquels se produisent la nucléation puis la croissance cristalline proprement dite de la phase cristallisée. En outre il permet de déterminer le point de première fusion du verre, donc la limite supérieure de température qu'il sera bon de ne pas dépasser afin d'éviter une déformation de la vitrocéramique due à la fusion des phases cristallisées. En outre il n'est pas exclu qu'un tel enregistrement apporte des informations sur la structure du verre. Cependant l'ATD ne doit pas être employée seule et il est nécessaire d'effectuer parallèlement des identifications par diffraction $\mathbf{X}$ ou des observations par microscopie optique ou électronique sur les phases cristallisées qui se forment lors du traitement thermique.

Nous allons décrire dans ce mémoire les résultats que nous avons obtenus dans l'étude de la dévitrification d'un certain nombre de verres synthétiques ou de matériaux vitreux (laitiers) appartenant aux systèmes $\mathrm{MgO}-\mathrm{Al}_{2} \mathrm{O}_{3}-\mathrm{SiO}_{2}, \quad \mathrm{MgO}-\mathrm{Al}_{2} \mathrm{O}_{3}-\mathrm{Fe}_{2} \mathrm{O}_{3}-\mathrm{SiO}_{2}$, $\mathrm{CaO}-\mathrm{Al}_{2} \mathrm{O}_{3}-\mathrm{SiO}_{2}, \mathrm{CaO}-\mathrm{MgO}-\mathrm{Al}_{2} \mathrm{O}_{3}-\mathrm{SiO}_{2}$ et

$$
\mathrm{CaO}-\mathrm{Fe}_{2} \mathrm{O}_{3}-\mathrm{Al}_{2} \mathrm{O}_{3}-\mathrm{SiO}_{2} \text {. }
$$

REVUE DE PHYSIQUe APPLIQUÉE. - T. 12 No 5, MAI 1977
2. Résultats expérimentaux et discussion. 2.1 VERRES DES SYSTÈMES $\mathrm{MgO}-\mathrm{Al}_{2} \mathrm{O}_{3}-\mathrm{SiO}_{2}$ ET $\mathrm{MgO}-\mathrm{Al}_{2} \mathrm{O}_{3}-\mathrm{Fe}_{2} \mathrm{O}_{3}-\mathrm{SiO}_{2}$. - Les verres du système $\mathrm{MgO}-\mathrm{Al}_{2} \mathrm{O}_{3}-\mathrm{SiO}_{2}$ et plus particulièrement les verres de cordiérite $2 \mathrm{MgO} .2 \quad \mathrm{Al}_{2} \mathrm{O}_{3} .5 \quad \mathrm{SiO}_{2}$ conduisent par dévitrification contrôlée à l'obtention de vitrocéramiques dont les propriétés (résistances mécaniques, coefficient de dilatation thermique, caractéristiques diélectriques) sont très intéressantes. Ces matériaux revêtent donc une grande importance du point de vue technologique.

Les différentes phases que l'on rencontre dans ce système sont les suivantes :

$\begin{array}{ll}\text { - } \begin{array}{l}\text { Silice (cristobalite et } \\ \text { tridymite) }\end{array} & \mathrm{SiO}_{2} \\ \text { - Protoenstatite } & \mathrm{MgO} \cdot \mathrm{SiO}_{2} \\ \text { - Forstérite } & 2 \mathrm{MgO} \cdot \mathrm{SiO}_{2} \\ \text { - Périclase } & \mathrm{MgO} \\ \text { - Spinelle } & \mathrm{MgO} \cdot \mathrm{Al}_{2} \mathrm{O}_{3} \\ \text { - Corindon } & \mathrm{Al}_{2} \mathrm{O}_{3} \\ \text { - Mullite } & 3 \mathrm{Al}_{2} \mathrm{O}_{3} \cdot \mathrm{SiO}_{2} \\ \text { - Cordiérite } & 2 \mathrm{MgO}_{2} \mathrm{Al}_{2} \mathrm{O}_{3} \cdot 5 \mathrm{SiO}_{2} \\ \text { - Saphir } & 4 \mathrm{MgO} .5 \mathrm{Al}_{2} \mathrm{O}_{3} \cdot 2 \mathrm{SiO}_{2} .\end{array}$

L'un d'entre nous [6] a utilisé l'ATD et la diffraction $X$ pour étudier six compositions de verre synthétisées dans ce système (Fig. 3) par fusion des mélanges d'oxydes puis trempe. Les températures auxquelles se manifeste la cristallisation thermique, qui se situe entre 900 et $1050^{\circ} \mathrm{C}$, sont assez variables selon les échantillons (Fig. 4). Il en est de même de la nature des phases qui cristallisent (Tableau I).

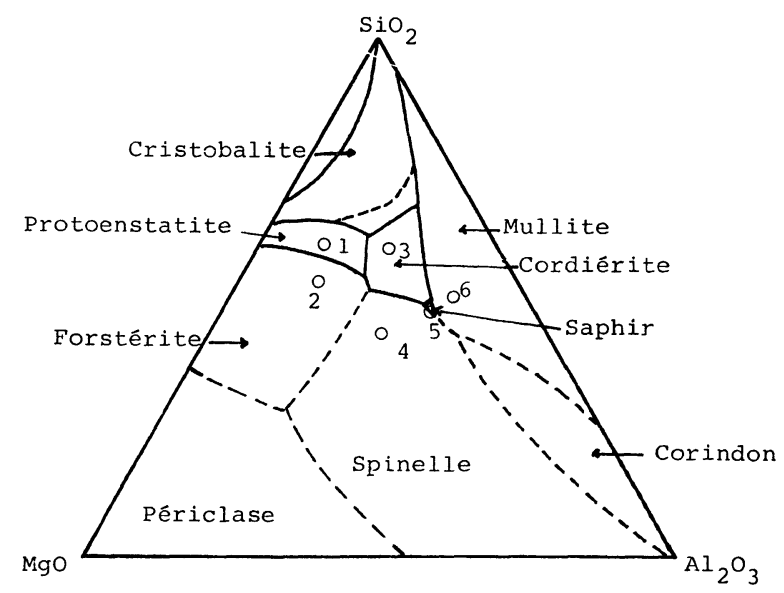

FIG. 3. - Position des compositions vitreuses (numérotées de 1 à 6) synthétisées dans le système $\mathrm{MgO}-\mathrm{Al}_{2} \mathrm{O}_{3}-\mathrm{SiO}_{2}$.

Deux observations ressortent de ces résultats :

- la température de cristallisation est d'autant plus basse que la teneur en $\mathrm{MgO}$ est plus importante ;

- la dévitrification se manifeste par un seul pic exothermique bien qu'elle conduise le plus souvent à 


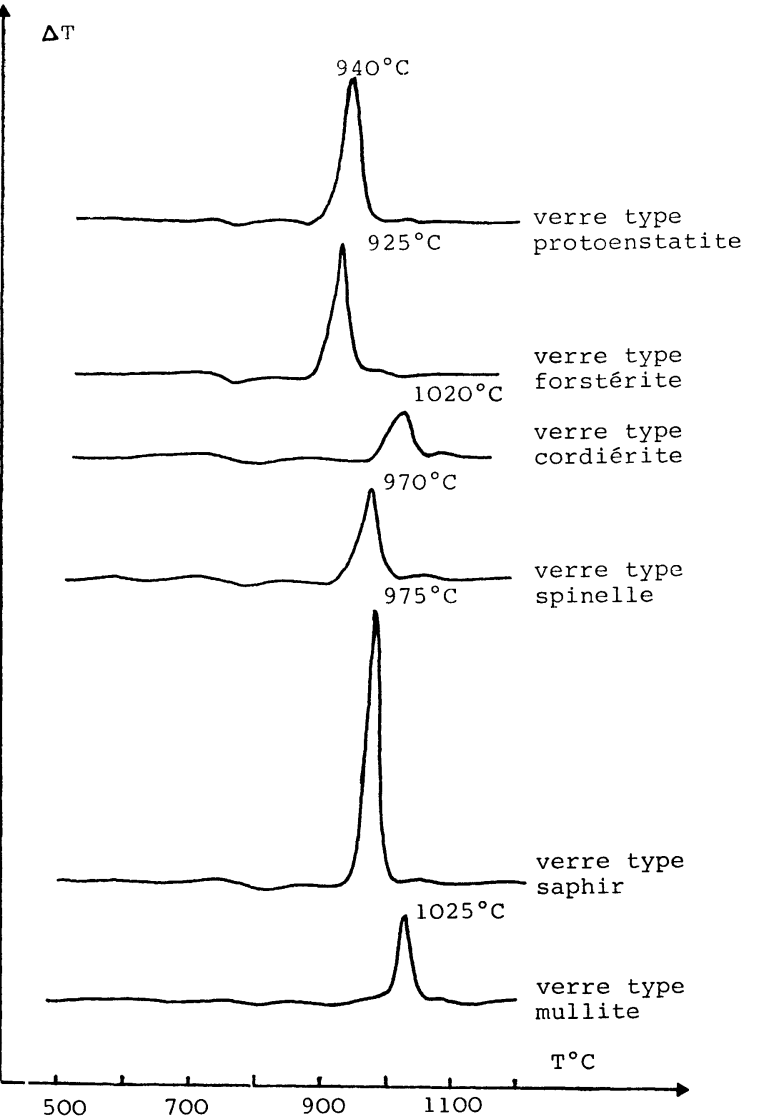

FIG. 4. - Courbes ATD des compositions vitreuses synthétisées dans le système $\mathrm{MgO}-\mathrm{Al}_{2} \mathrm{O}_{3}-\mathrm{SiO}_{2}$.

un mélange de phases ne correspondant pas forcément au domaine relatif à la composition qui avait été fixée au départ, mais le plus souvent aux domaines voisins (Fig. 3).
2.1.1 Etude particulière du verre de cordiérite. Le verre de cordiérite pure $\left(2 \mathrm{MgO} .2 \mathrm{Al}_{2} \mathrm{O}_{3} .5 \mathrm{SiO}_{2}\right)$ présente un pic exothermique de dévitrification dont le maximum se situe à $1005^{\circ} \mathrm{C}$. Il se forme de la cordiérite $\alpha$. Ce pic est précédé d'une déflexion exothermique très marquée entre 700 et $900^{\circ} \mathrm{C}$ (Fig. 5).

La nucléation peut être contrôlée par addition au verre (au moment de la synthèse ou après trempe et

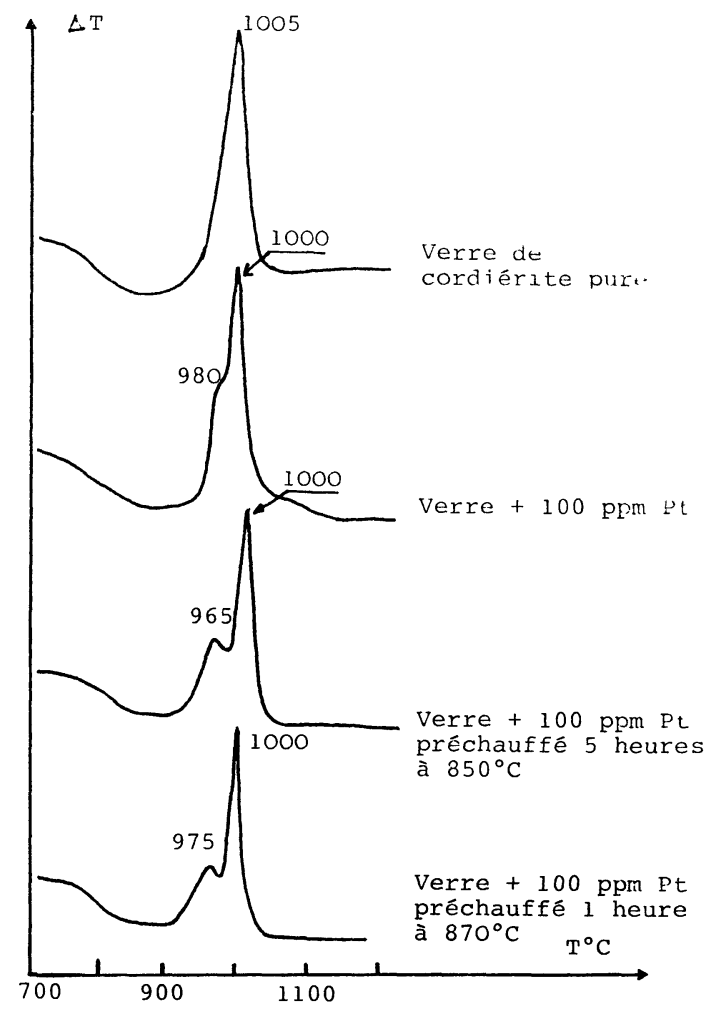

Fig. 5. - Courbes ATD de cordiérite pure et de cordiérite contenant $100 \mathrm{ppm}$. de platine préchauffée ou non.

\section{TABleAU I}

\begin{tabular}{|c|c|c|c|c|c|c|c|c|c|c|c|c|}
\hline \multirow[b]{2}{*}{$\begin{array}{c}\text { Composition chimique } \\
(\%)\end{array}$} & \multicolumn{12}{|c|}{ Domaines de composition étudiée } \\
\hline & \multicolumn{2}{|c|}{$\begin{array}{c}1 \\
\text { Protoens. }\end{array}$} & \multicolumn{2}{|c|}{$\begin{array}{l}2 \\
\text { Forst. }\end{array}$} & \multicolumn{2}{|c|}{$\begin{array}{c}3 \\
\text { Cord. }\end{array}$} & \multicolumn{2}{|c|}{$\begin{array}{c}4 \\
\text { Spin. }\end{array}$} & \multicolumn{2}{|c|}{$\begin{array}{c}5 \\
\text { Saph. }\end{array}$} & \multicolumn{2}{|c|}{$\stackrel{6}{6}$} \\
\hline $\mathrm{SiO}_{2}$ & \multicolumn{2}{|c|}{61,0} & \multicolumn{2}{|c|}{53,0} & \multicolumn{2}{|c|}{59,9} & \multicolumn{2}{|c|}{53,7} & \multicolumn{2}{|c|}{47,9} & \multicolumn{2}{|c|}{50,0} \\
\hline $\mathrm{Al}_{2} \mathrm{O}_{3}$ & \multicolumn{2}{|c|}{10,3} & \multicolumn{2}{|c|}{10,9} & \multicolumn{2}{|c|}{23,3} & \multicolumn{2}{|c|}{28,9} & \multicolumn{2}{|c|}{35,1} & \multicolumn{2}{|c|}{37,9} \\
\hline $\mathrm{MgO}$ & \multicolumn{2}{|c|}{28,7} & \multicolumn{2}{|c|}{36,1} & \multicolumn{2}{|c|}{16,8} & \multicolumn{2}{|c|}{27,4} & \multicolumn{2}{|c|}{17,0} & \multicolumn{2}{|c|}{12,1} \\
\hline$T_{\mathrm{m}}\left({ }^{\circ} \mathrm{C}\right) \mathrm{ATD}$ & \multicolumn{2}{|c|}{940} & \multicolumn{2}{|c|}{925} & \multicolumn{2}{|c|}{1020} & \multicolumn{2}{|c|}{970} & \multicolumn{2}{|c|}{975} & \multicolumn{2}{|c|}{1025} \\
\hline $\begin{array}{l}\text { Nature des phases cristal- } \\
\text { lisées formées }(*)\end{array}$ & (a) & (b) & (a) & (b) & (a) & (b) & (a) & (b) & (a) & (b) & (a) & (b) \\
\hline Enstatite & $\mathrm{m}$ & $\mathrm{m}$ & $\mathrm{f}$ & f & f & $\mathrm{f}$ & $\mathrm{m}$ & $\mathrm{m}$ & & & & \\
\hline Forstérite & & & f & & & & $\mathrm{f}$ & f & & & & \\
\hline Cordiérite $\mu$ & & & F & $\mathrm{F}$ & $\mathrm{F}$ & $\mathrm{F}$ & F & $\mathrm{m}$ & $\mathrm{f}$ & $\mathrm{f}$ & F & $\mathrm{F}$ \\
\hline Cordiérite $\alpha$ & & & & $\mathrm{m}$ & & $\mathrm{f}$ & & $\mathrm{F}$ & $\mathrm{F}$ & $\mathrm{F}$ & $\mathbf{F}$ & $\mathrm{F}$ \\
\hline Mullite & & & & & & & & & & & $\mathrm{f}$ & $\mathrm{f}$ \\
\hline
\end{tabular}

L'intensité des raies est caractérisée par la lettre $F$ (forte), $m$ (moyenne) et $\mathrm{f}$ (faible).

(*) L'identification par diffraction X est réalisée sur les échantillons maintenus - soit 1/4 d'heure (colonne (a)), - soit 10 heures (colonne (b)) à la température $T_{\mathrm{m}}$ du sommet du pic ATD. 
broyage) de certaines substances dénommées agents de nucléation.

On peut ainsi utiliser le platine qui est ajouté sous forme d'acide chloroplatinique au mélange d'oxydes de départ avant fusion. On peut également mélanger certains oxydes $\left(\mathrm{Al}_{2} \mathrm{O}_{3}, \mathrm{ZrO}_{2}, \mathrm{Cr}_{2} \mathrm{O}_{3}, \mathrm{TiO}_{2}\right.$, etc...) au verre broyé après trempe.

On assiste alors à une évolution caractéristique des courbes ATD selon l'agent de nucléation employé et la teneur en celui-ci.

Ainsi, l'incorporation de $100 \mathrm{ppm}$. de platine [7] au verre de cordiérite conduit à un épaulement caractéristique à $980^{\circ} \mathrm{C}$ sur le pic de dévitrification (Fig. 5). Cet épaulement se transforme en un véritable pic de dévitrification lorsque le verre est préalablement recuit pendant 5 heures à $850^{\circ} \mathrm{C}$ ou seulement une heure à $870^{\circ} \mathrm{C}$. Ce pic traduit la cristallisation de cordiérite $\mu$ métastable, dont la nucléation est provoquée par le platine lors des opérations de recuit.

La formation de cordiérite $\mu$ (métastable) se manifeste également lorsque l'on ajoute au verre des oxydes métalliques tels que ceux mentionnés plus haut. Dans ce cas les quantités d'oxyde ajoutées doivent être déjà assez importantes pour que le dédoublement du pic ATD soit observable (Fig. 6).

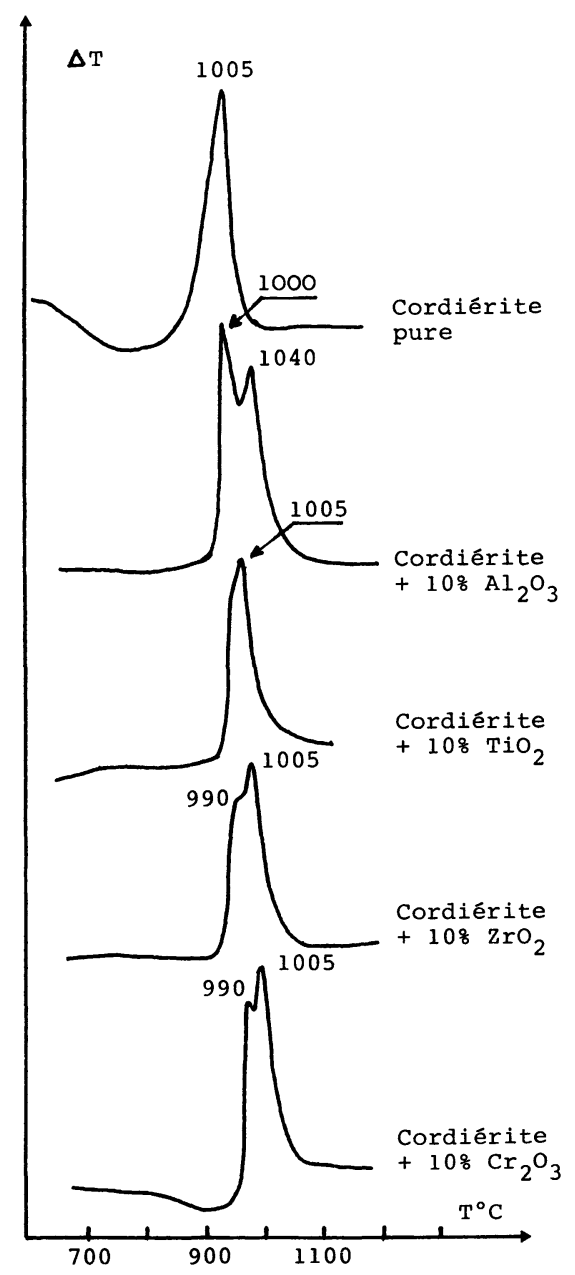

FIG. 6. - Courbes ATD de cordiérite pure et de cordiérite additionnée de $10 \%$ (en poids) de différents oxydes métalliques.
Dans le cas de l'addition de $\mathrm{TiO}_{2}, \mathrm{ZrO}_{2}$ [8] et $\mathrm{Cr}_{2} \mathrm{O}_{3}$ [9] la cordiérite $\mu$ apparaît systématiquement avant la cordiérite $\alpha$, quelle que soit la quantité d'oxyde ajoutée entre 10 et $90 \%$. A température plus élevée se forment la cordiérite $\alpha$, le titanate de magnésium, la cristobalite, et une phase spinelle.

Dans le cas de l'addition d'alumine le pic à $1000^{\circ} \mathrm{C}$ (Fig. 6) correspond à la cristallisation de la cordiérite $\mu$, celui à $1040^{\circ} \mathrm{C}$ à la formation de cordiérite $\alpha$. Pour des additions d'alumine $\geqslant 60 \%$ seul reste un pic exothermique à $980^{\circ} \mathrm{C}$ correspondant à la cristallisation de la cordiérite $\mu$. L'addition de quantités importantes d'alumine stabilise donc cette phase [10].

\subsubsection{Etude de verres de cordiérite substituée en} fer. - L'étude par ATD et radiocristallographie de la recristallisation thermique de verres de cordiérite contenant du fer III a permis de mettre en évidence l'influence de ce cation sur le phénomène de dévitrification [11]. Les compositions suivantes ont été synthétisées par fusion des mélanges d'oxydes puis trempe :

$$
\begin{aligned}
& 1: 2 \mathrm{MgO} .2 \mathrm{Al}_{2} \mathrm{O}_{3} \cdot 5 \mathrm{SiO}_{2} \\
& 2: 2 \mathrm{MgO} .1,75 \mathrm{Al}_{2} \mathrm{O}_{3} \cdot 0,25 \mathrm{Fe}_{2} \mathrm{O}_{3} \cdot 5 \mathrm{SiO}_{2} \\
& 3: 2 \mathrm{MgO} .1,50 \mathrm{Al}_{2} \mathrm{O}_{3} \cdot 0,50 \mathrm{Fe}_{2} \mathrm{O}_{3} \cdot 5 \mathrm{SiO}_{2} \\
& 4: 2 \mathrm{MgO} . \mathrm{Al}_{2} \mathrm{O}_{3} \cdot \mathrm{Fe}_{2} \mathrm{O}_{3} \cdot 5 \mathrm{SiO}_{2} \\
& 5: 2 \mathrm{MgO} .0,50 \mathrm{Al}_{2} \mathrm{O}_{3} \cdot 1,50 \mathrm{Fe}_{2} \mathrm{O}_{3} \cdot 5 \mathrm{SiO}_{2} \\
& 6: 2 \mathrm{MgO} .2 \mathrm{Fe}_{2} \mathrm{O}_{3} \cdot 5 \mathrm{SiO}_{2}
\end{aligned}
$$

Les courbes ATD manifestent une évolution très spectaculaire (Fig. 7). Le tableau II indique la nature des phases obtenues après dévitrification.

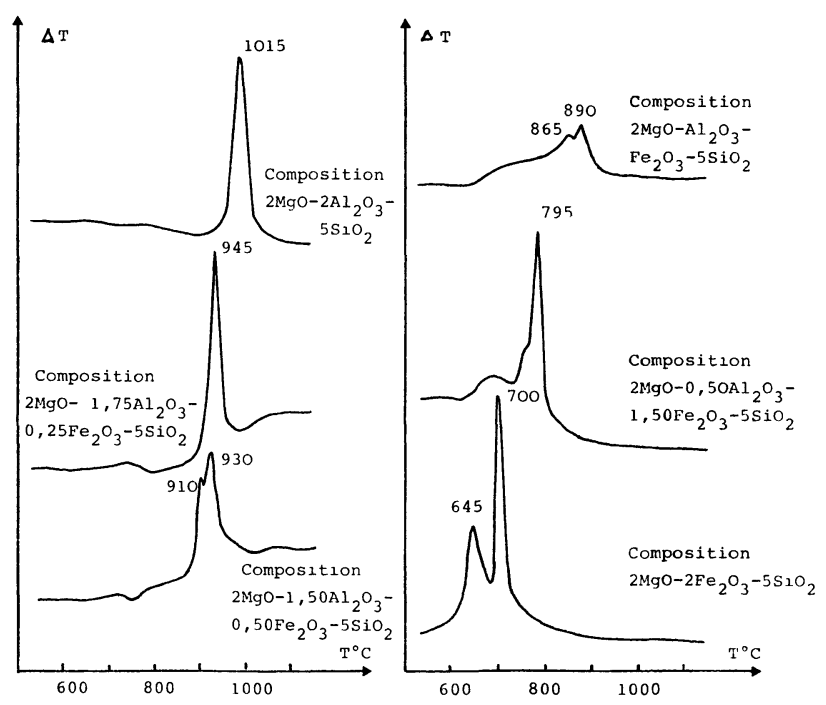


$\mathrm{Fe}_{2} \mathrm{O}_{3}-\mathrm{SiO}_{2}$.

On note une différence très nette de part et d'autre de la composition

$$
2 \mathrm{MgO} .1,5 \mathrm{Al}_{2} \mathrm{O}_{3} \cdot 0,50 \mathrm{Fe}_{2} \mathrm{O}_{3} \cdot 5 \mathrm{SiO}_{2} \text {. }
$$

Pour les verres les moins riches en fer la recristallisation conduit simultanément à de la cordiérite $\mu$ et de la 


\section{TABLEAU II}

Composition

\begin{tabular}{|c|c|c|}
\hline \multicolumn{2}{|c|}{$\begin{array}{l}\text { Composition } \\
\text { nombre de moles }\end{array}$} & $\begin{array}{c}T_{\mathrm{m}}\left({ }^{\circ} \mathrm{C}\right. \\
\text { ATD }\end{array}$ \\
\hline 2 & 0 & 1015 \\
\hline 1,75 & 0,25 & 945 \\
\hline 1,50 & 0,50 & $\begin{array}{l}910 \\
930\end{array}$ \\
\hline 1,0 & 1,0 & 865 \\
\hline 0,50 & 1,50 & 795 \\
\hline 0 & 2,0 & $\begin{array}{l}640 \\
700\end{array}$ \\
\hline
\end{tabular}

Nature des phases cristallisées formées

Cord. $\mu$
-
f
f
f

cordiérite $\alpha$ avec dédoublement du pic ATD pour la composition 3. A partir de la composition 4 les phases qui cristallisent sont le spinelle $\mathrm{MgO} .\left(\mathrm{Fe}_{x} \mathrm{Al}_{2-x}\right) \mathrm{O}_{3}$, l'enstatite $\mathrm{MgO} . \mathrm{SiO}_{2}$ et les oxydes de fer $\left(\mathrm{Fe}_{2} \mathrm{O}_{3} \alpha\right.$, $\left.\mathrm{Fe}_{3} \mathrm{O}_{4}\right)$. La cordiérite totalement substituée en fer $2 \mathrm{MgO} .2 \mathrm{Fe}_{2} \mathrm{O}_{3} .5 \mathrm{SiO}_{2}$ donne deux pics de dévitrification situés à des températures bien plus basses que celle relative à la cordiérite normale.

Cette étude montre que le fer a une influence marquée sur le processus et la température de dévitrification.

Ces résultats sont à rapprocher de ceux publiés récemment par Sosulja et Wachtler [12] à propos de la température de cristallisation thermique de verres dans le système $\mathrm{CaO} \cdot \mathrm{Al}_{2} \mathrm{O}_{3} \cdot \mathrm{Fe}_{2} \mathrm{O}_{3}$. Les courbes ATD montrent que la température de début et du maximum de l'effet exothermique est d'autant plus basse que la teneur en fer est plus élevée (Tableau III). On constate parallèlement l'augmentation de la température de cristallisation avec l'accroissement de la teneur en alumine.

2.2 Verres DeS SYStèmes $\mathrm{CaO} \cdot \mathrm{Al}_{2} \mathrm{O}_{3} \cdot \mathrm{SiO}_{2}$ ET $\mathrm{CaO} . \mathrm{Al}_{2} \mathrm{O}_{3} \cdot \mathrm{MgO} \cdot \mathrm{SiO}_{2}$. ETUde DE LAITIERS INDUSTRIELS. - Les laitiers ou scories sont les sous-produits de la fabrication et du raffinage de la fonte et de très nombreuses opérations de pyro- ou d'électrométallurgie. Ces matériaux sont constitués généralement de silice, d'alumine, et de chaux mais peuvent contenir des quantités non négligeables d'oxydes de fer, de

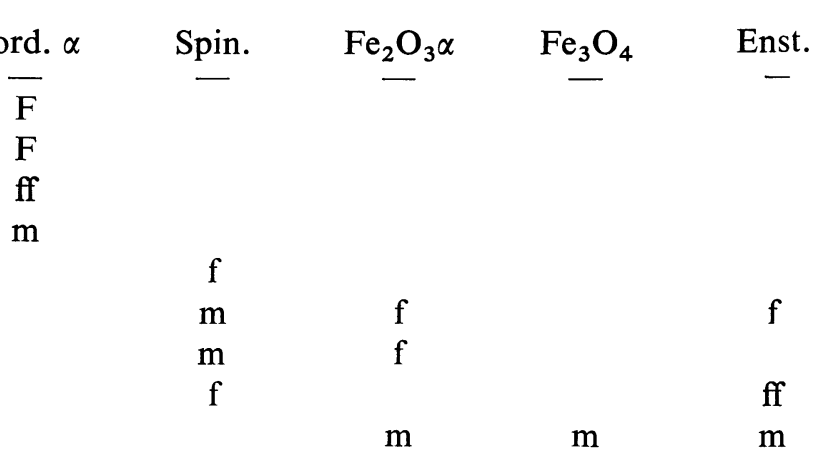

magnésie, ou d'oxydes d'autres métaux, des sulfures, et des alcalins en petites quantités.

Suivant le mode de refroidissement ces matériaux peuvent se présenter soit à l'état cristallisé, soit à l'état vitreux ou partiellement vitrifié.

Les laitiers granulés de haut-fourneau sont généralement vitreux (laitiers granulés, sables de laitier) et ont trouvé depuis longtemps une utilisation comme matière première en chimie des ciments. Depuis une vingtaine d'années on a pensé à les utiliser comme matière première de substitution dans la fabrication des vitrocéramiques [13] ou des verres [14]. C'est ces derniers types d'utilisation qui nous intéressent plus particulièrement.

L'état de vitrification des laitiers peut être caractérisé par différentes méthodes :

- aspect visuel de verre avec cassure conchoïdale ;

- couleur de fluorescence allant du rose pour les éléments vitreux, au bleu-noir pour les composés cristallisés, lorsque l'échantillon est soumis à un rayonnement UV ;

- essai dit de recalescence qui constitue à enregistrer la courbe d'analyse thermique simple ou d'ATD du laitier, courbes qui présentent un crochet pour la première, un pic pour la seconde, phénomène dont l'intensité renseigne sur l'état de vitrification du matériau ;

- mesure de la chaleur de recristallisation qui est la différence entre la chaleur de dissolution dans $\mathrm{HNO}_{3}$

\section{TABLEAU III}

\begin{tabular}{|c|c|c|c|c|c|c|}
\hline Compositions & $\begin{array}{l}\mathrm{Fe}_{2} \mathrm{O}_{3} \\
\% \text { masse }\end{array}$ & $\begin{array}{c}\mathrm{Fe}_{2} \mathrm{O}_{3} \\
\% \text { moles }\end{array}$ & $\begin{array}{c}\mathrm{Al}_{2} \mathrm{O}_{3} \\
\% \text { masse }\end{array}$ & $\begin{array}{c}\mathrm{Al}_{2} \mathrm{O}_{3} \\
\% \text { moles }\end{array}$ & $T_{\text {début }}$ & $T_{\max }$ \\
\hline $2 \mathrm{CaO} \cdot \overline{\mathrm{Fe}}_{2} \mathrm{O}_{3}$ & 58,8 & 33,3 & 0 & 0 & 380 & $49 \overline{(*)}$ \\
\hline $6 \mathrm{CaO} \cdot \mathrm{Al}_{2} \mathrm{O}_{3} \cdot 2 \mathrm{Fe}_{2} \mathrm{O}_{3}$ & 42,2 & 22,5 & 13,45 & 11,2 & 390 & $520(*)$ \\
\hline $4 \mathrm{CaO} \cdot \mathrm{Al}_{2} \mathrm{O}_{3} \cdot \mathrm{Fe}_{2} \mathrm{O}_{3}$ & 32,9 & 16,6 & 20,48 & 16,6 & 690 & 750 \\
\hline $6 \mathrm{CaO} .2 \mathrm{Al}_{2} \mathrm{O}_{3} \cdot \mathrm{Fe}_{2} \mathrm{O}_{3}$ & 22,8 & 11,2 & 29,14 & 22,5 & 740 & 780 \\
\hline $8 \mathrm{CaO} .3 \mathrm{Al}_{2} \mathrm{O}_{3} \cdot \mathrm{Fe}_{2} \mathrm{O}_{3}$ & 17,5 & 8,3 & 33,47 & 25,0 & 750 & 790 \\
\hline $12 \mathrm{CaO} .7 \mathrm{Al}_{2} \mathrm{O}_{3}$ & 0 & 0 & 51,55 & 35,8 & 850 & 940 \\
\hline
\end{tabular}

(*) Réactions lentes : le pic exothermique est très aplati et peu intense. 
ou HF d'un laitier donné et du même échantillon dévitrifié par chauffage à $1000^{\circ} \mathrm{C}$ par exemple. Cette chaleur varie entre 40 et $80 \mathrm{cal} / \mathrm{g}$ pour les laitiers ;

- étude par diffraction $X$ pouvant donner un pourcentage de phase cristallisée ;

- observation par microscopie optique après attaque de l'échantillon par des réactifs appropriés.

De nombreux auteurs [15, 19] ont utilisé l'ATD aux fins de caractérisation des laitiers de haut-fourneau. Mais les avis restent encore assez partagés quant à l'interprétation des résultats. En effet, chaque laitier présente sa courbe d'ATD caractéristique, d'allure très variable d'un échantillon à un autre et très sensible aux fluctuations et hétérogénéités de composition. Ces recherches ont été axées essentiellement sur l'établissement de corrélations entre l'allure des courbes ATD et l'hydraulicité du produit (réactivité lorsqu'il est soumis à hydratation). Un certain nombre de conclusions a été tiré de ces travaux :

- la nature et la quantité de phases cristallines formées lors du chauffage (dévitrification) n'a pas de rapport avec le pouvoir de durcissement hydraulique du matériau;

- les laitiers dont les courbes ATD présentent plusieurs pics exothermiques ont généralement un rapport $\mathrm{CaO} / \mathrm{SiO}_{2}$ élevé ;

- il existe une certaine corrélation entre la température du maximum du pic exothermique de cristallisation et la valeur du rapport $\mathrm{SiO}_{2} / \mathrm{Al}_{2} \mathrm{O}_{3}$.

Par ailleurs, la présence de plusieurs pics d'ATD dans le cas de laitiers vitreux broyés est associée à une dévitrification en plusieurs étapes : certains grains se dévitrifient les premiers, les autres ne cristallisent qu'à une température plus élevée. Ces observations ont été confirmées par microscopie optique et par diffraction sur des grains refroidis après la manifestation de chaque effet exothermique [19].

Les principales phases qui cristallisent lors du chauffage des laitiers vitreux de haut-fourneau sont les suivantes $\left({ }^{1}\right)$ :

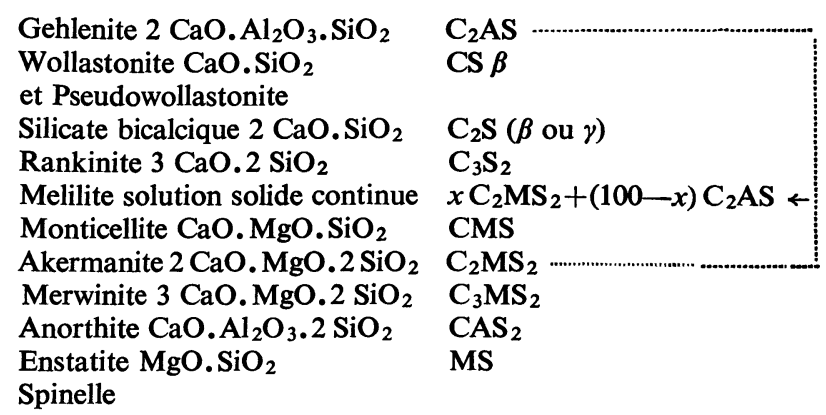

Il existe également la mélilite ferrique dans laquelle une partie de l'alumine peut être substituée par $\mathrm{Fe}_{2} \mathrm{O}_{3}$.

Dans le but de fabriquer des vitrocéramiques en utilisant les laitiers comme matière première de substitution, nous avons étudié récemment, à l'aide des techniques ATD, de diffraction $\mathrm{X}$, de dilatométrie, et de microscopie électronique à balayage [20] une série de laitiers industriels qui diffèrent par leur morphologie [21-22] et leur composition (Tableau IV).

Comme les autres auteurs qui avaient abordé ce problème, nous avons constaté que les courbes ATD (Fig. 8) étaient très variables d'un échantillon à un autre.

On observe généralement un ou plusieurs pics exothermiques, d'intensité et de forme variables. Parfois ce pic ne correspond à aucune cristallisation comme c'est le cas pour l'échantillon I.5 (pic à $465^{\circ} \mathrm{C}$ ) et pour I.6.

(1) $\mathrm{C}=\mathrm{CaO} ; \mathrm{S}=\mathrm{SiO}_{2} ; \mathrm{A}=\mathrm{Al}_{2} \mathrm{O}_{3} ; \mathrm{M}=\mathrm{MgO}$ écriture généralement adoptée pour les silicates.

TABLEAU IV

Composition des laitiers étudiés

\begin{tabular}{|c|c|c|c|c|c|c|c|c|}
\hline Echantillons & $\mathrm{CaO}$ & $\mathrm{SiO}_{2}$ & $\mathrm{Al}_{2} \mathrm{O}_{3}$ & $\mathrm{MgO}$ & $\mathrm{Fe}_{2} \mathrm{O}_{3}$ & $\mathrm{Na}_{2} \mathrm{O}$ & $\mathrm{K}_{2} \mathrm{O}$ & Insol. \\
\hline F.1N & 39,77 & 41,77 & 12,93 & 1,15 & 2,43 & 0,63 & 1,07 & 0,09 \\
\hline F.1BR & 43,05 & 43,07 & 8,36 & 1,10 & 2,80 & 0,50 & 0,80 & 0,15 \\
\hline F.1BL & 46,44 & 42,01 & 7,44 & 1,12 & 1,77 & 0,60 & 0,93 & 0,08 \\
\hline I. 1 & 41,96 & 31,39 & 11,75 & 12,11 & 2,00 & 0,22 & 0,30 & 0,41 \\
\hline 1.2 & 37,35 & 48,77 & 9,02 & 0,17 & 3,17 & 0,18 & 0,43 & 0,44 \\
\hline I. 3 & 30,82 & 38,74 & 25,75 & 0,29 & 1,22 & 0,21 & 1,08 & 1,78 \\
\hline I. 4 & 33,72 & 34,45 & 8,00 & 18,36 & 3,71 & 0,13 & 0,36 & 0,53 \\
\hline I. $5(*)$ & 26,35 & 39,40 & 19,49 & 11,05 & 0,42 & 0,15 & 1,02 & 0,37 \\
\hline I. 6 & 41,15 & 33,00 & 14,15 & 7,51 & 0,98 & 0,66 & 1,22 & 0,61 \\
\hline I. 7 & 41,87 & 35,54 & 18,49 & 0,12 & 2,39 & 0,49 & 0,44 & 0,25 \\
\hline I. 8 & 37,91 & 48,11 & 11,67 & 0,11 & 0,79 & 0,11 & 0,35 & 0,41 \\
\hline I. 9 & 38,82 & 35,24 & 12,25 & 10,60 & 1,51 & 0,20 & 0,28 & 0,57 \\
\hline E. 1 & 39,88 & 37,89 & 17,27 & 0,11 & 0,77 & 0,52 & 1,62 & 1,74 \\
\hline
\end{tabular}

(*) Le laitier I. 5 contient en outre environ 2,1\% de MnO. 

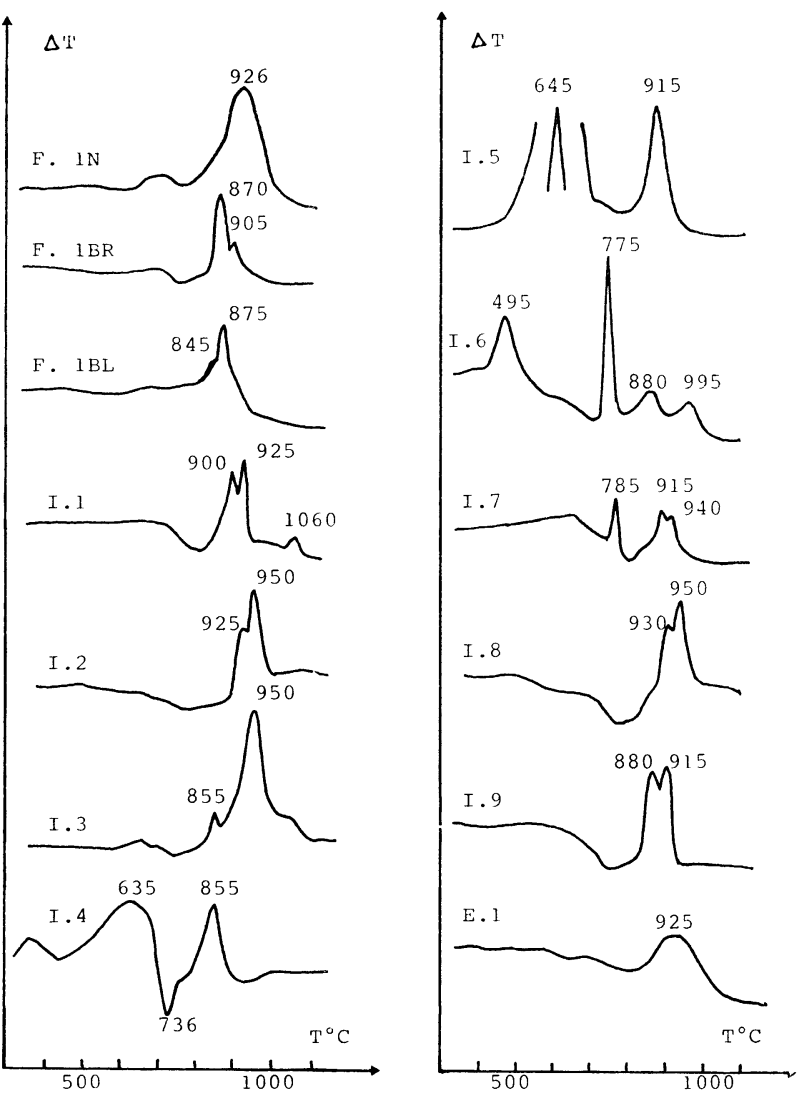

FIG. 8. - Courbes ATD de laitiers industriels.

On note également une déflexion endothermique très marquée avant la manifestation du premier effet exothermique. Ce phénomène a été attribué selon les auteurs - soit à l'absorption par le matériau vitreux, d'une certaine quantité d'énergie conférant aux ions la mobilité nécessaire à l'établissement de la structure cristalline $[16,17]$ donc à la nucléation de la phase cristallisée [12] - soit à un frittage du solide [16].

Une étude par dilatométrie sur poudre compactée nous a montré qu'à cette déflexion endothermique était associée une contraction dont l'intensité était très variable selon le laitier soumis à l'essai (Fig. 9, Fig. 10). Des observations par microscopie électronique à balayage sur le matériau chauffé soit avant $\left(700^{\circ} \mathrm{C}\right)$ soit en fin de contraction $\left(830-850^{\circ} \mathrm{C}\right)$, associées à des analyses par diffraction $X$ nous ont montré que ce phénomène était complexe :

- quand la contraction est faible $(\Delta L / L \simeq 1 \%)$ (cas de l'échantillon I.1) la morphologie des grains ne change pas (Fig. 11) et l'analyse par diffraction $\mathrm{X}$ montre que l'on est en tout début de cristallisation;

- quand la contraction est forte $(\Delta L / L \geqslant 15 \%)$ deux cas se présentent : soit un début de frittage de la poudre (Fig. 12) auquel se superpose l'apparition d'une phase cristallisée, soit aucune formation de phase cristallisée mais un frittage intensif du matériau (Fig. 13), la contraction étant alors plus importante $(\Delta L / L>25 \%)$.

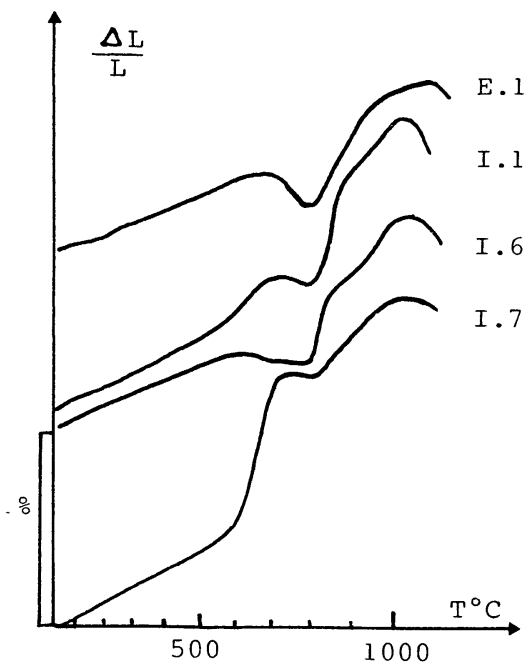

Fig. 9. - Courbes dilatométriques de laitiers industriels.

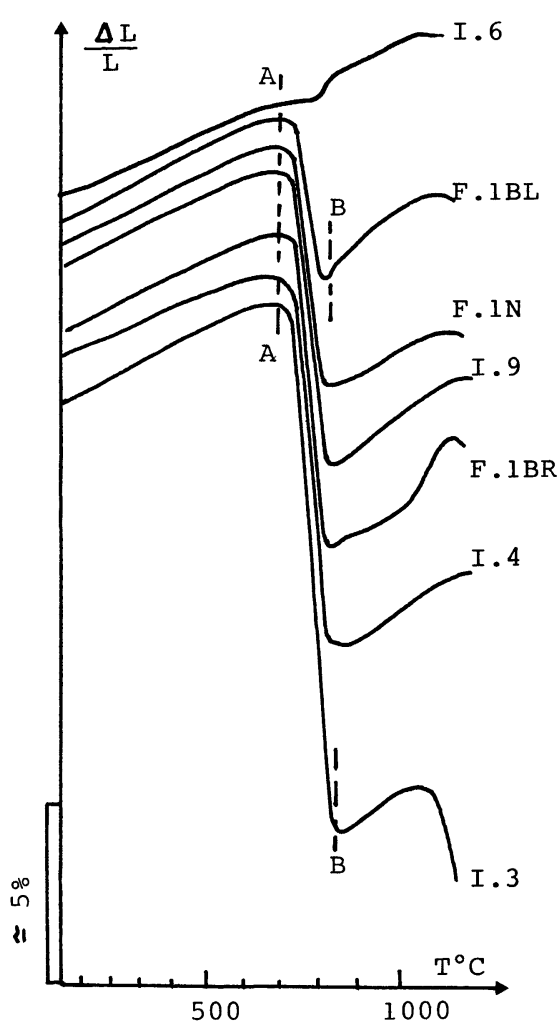

FIG. 10. - Courbes dilatométriques de laitiers industriels.

Par ailleurs la nature des phases cristallisées qui se forment lors de la dévitrification varie selon les échantillons (tableau V) : on observe en particulier que dans le cas de solides à teneur notable en $\mathrm{MgO}$ la première phase qui cristallise est systématiquement la merwinite $3 \mathrm{CaO} . \mathrm{MgO} .2 \mathrm{SiO}_{2}$.

Compte tenu de la complexité de ces phénomènes et du fait qu'aucune corrélation ne peut être établie entre les températures de recristallisation thermique et la composition des échantillons, nous avons été amenés à travailler sur des matériaux plus simples, à savoir : des laitiers synthétiques, préparés au Laboratoire, et 
Laitier I-1. $700{ }^{\circ} \mathrm{C}(\times 2500)$
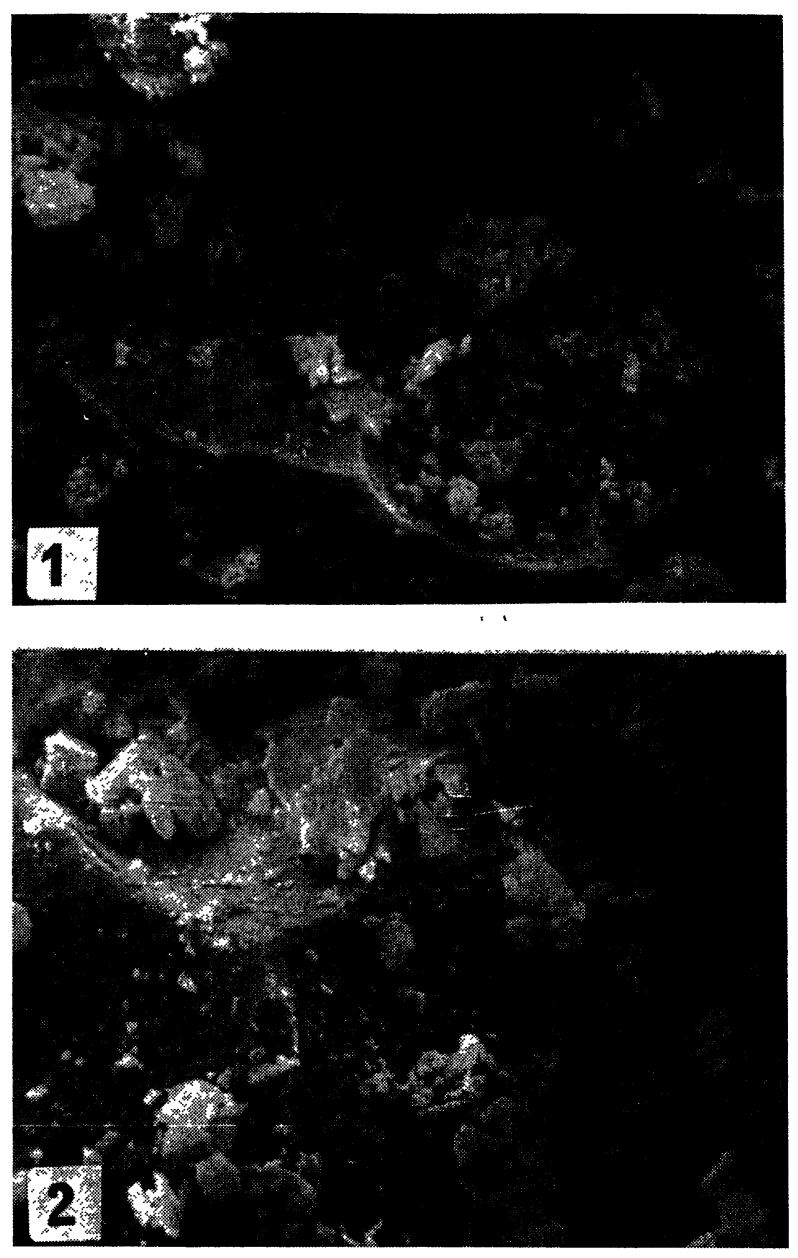

Laitier I-1. $830^{\circ} \mathrm{C}(\times 2500)$

Fig. 11. - Clichés de Meb. d'un laitier (échantillon I.1) chauffé à $700^{\circ} \mathrm{C}$ (cliché $1 ; \times 2500$ ) et à $830^{\circ} \mathrm{C}$ (cliché $2 ; \times 2500$ ).

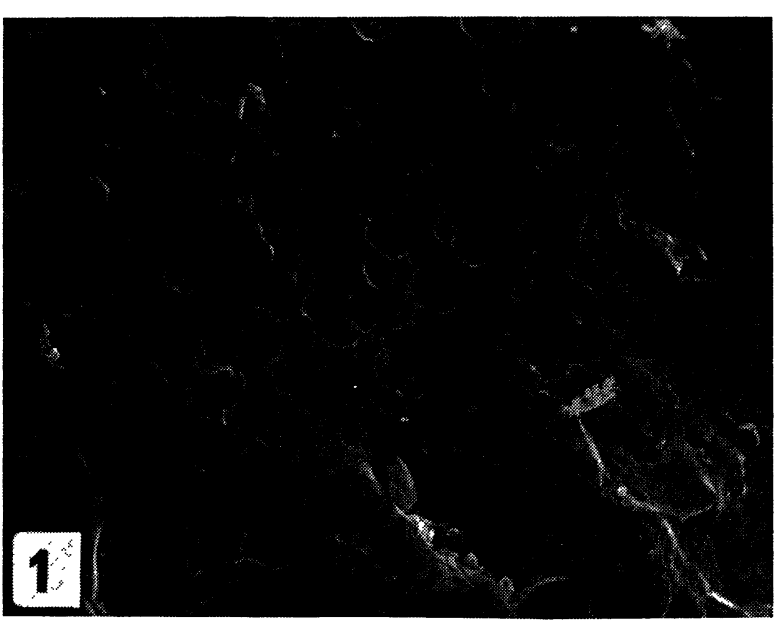

Laitier I-2. $830^{\circ} \mathrm{C}(\times 500)$
Laitier I-3. $830{ }^{\circ} \mathrm{C}(\times 2000)$
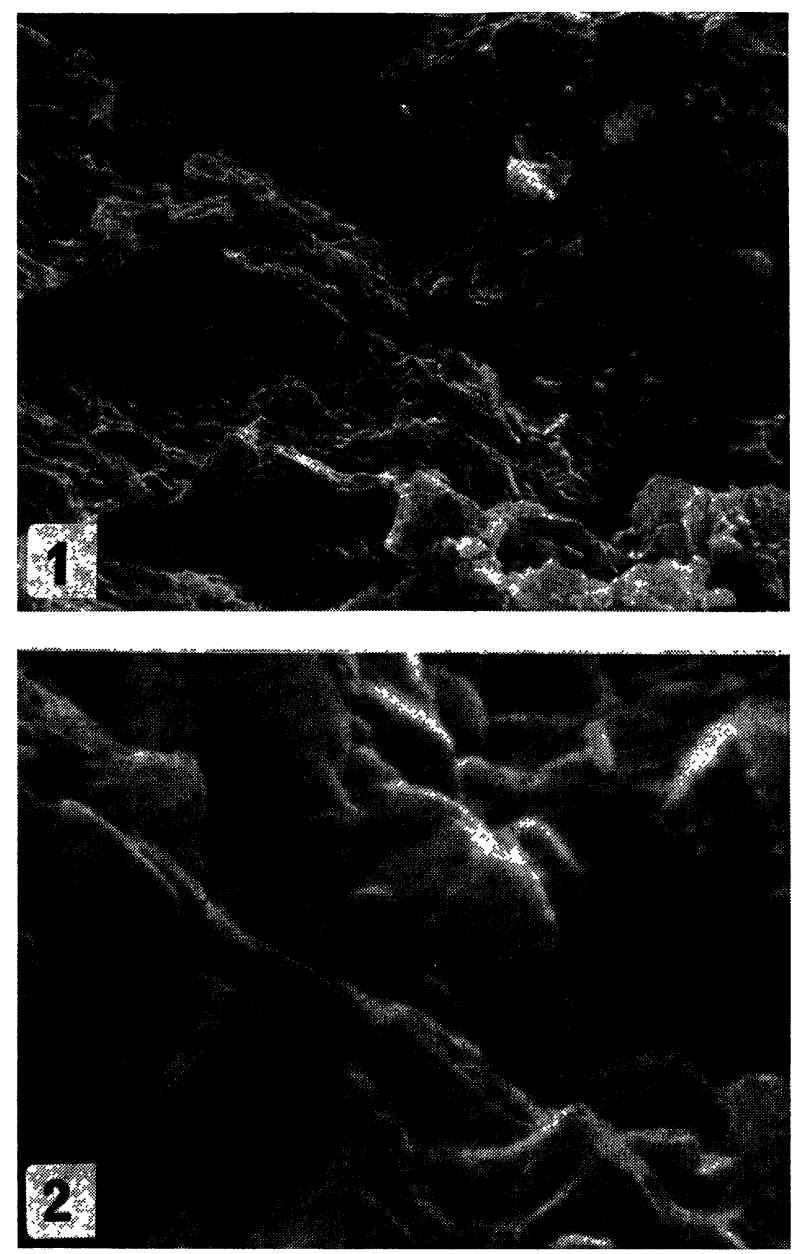

Laitier I-3. $830{ }^{\circ} \mathrm{C}(\times 10000)$

Fig. 12. - Clichés de Meb. d'un laitier (échantillon I.3) chauffé à $830^{\circ} \mathrm{C}$ (cliché 1: $\times 2000$; cliché $\left.2: \times 10000\right)$.

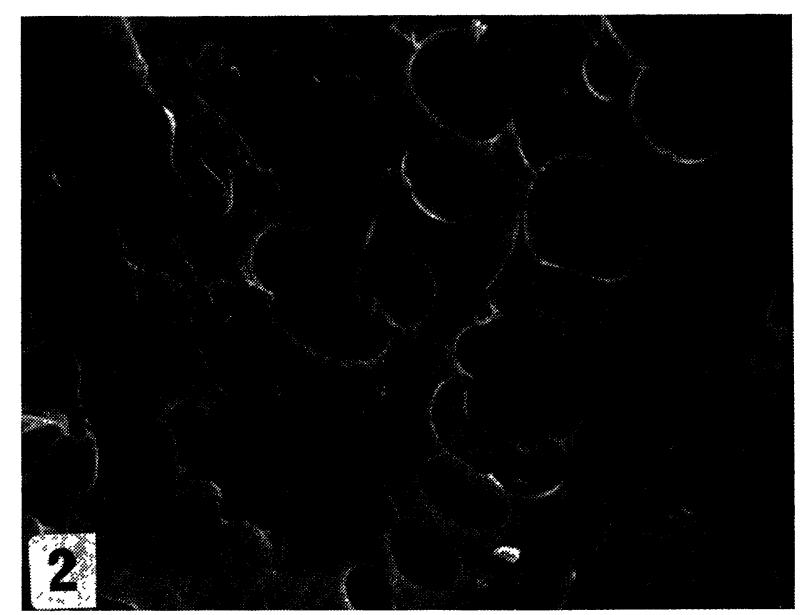

Laitier I-2. $830^{\circ} \mathrm{C}(\times 1000)$

FIG. 13. - Clichés de Meb. d'un laitier (échantillon I.2) chauffé à $830^{\circ} \mathrm{C}$ (cliché $1: \times 500$; cliché $2: \times 1000$ ). 


\section{TABleau V}

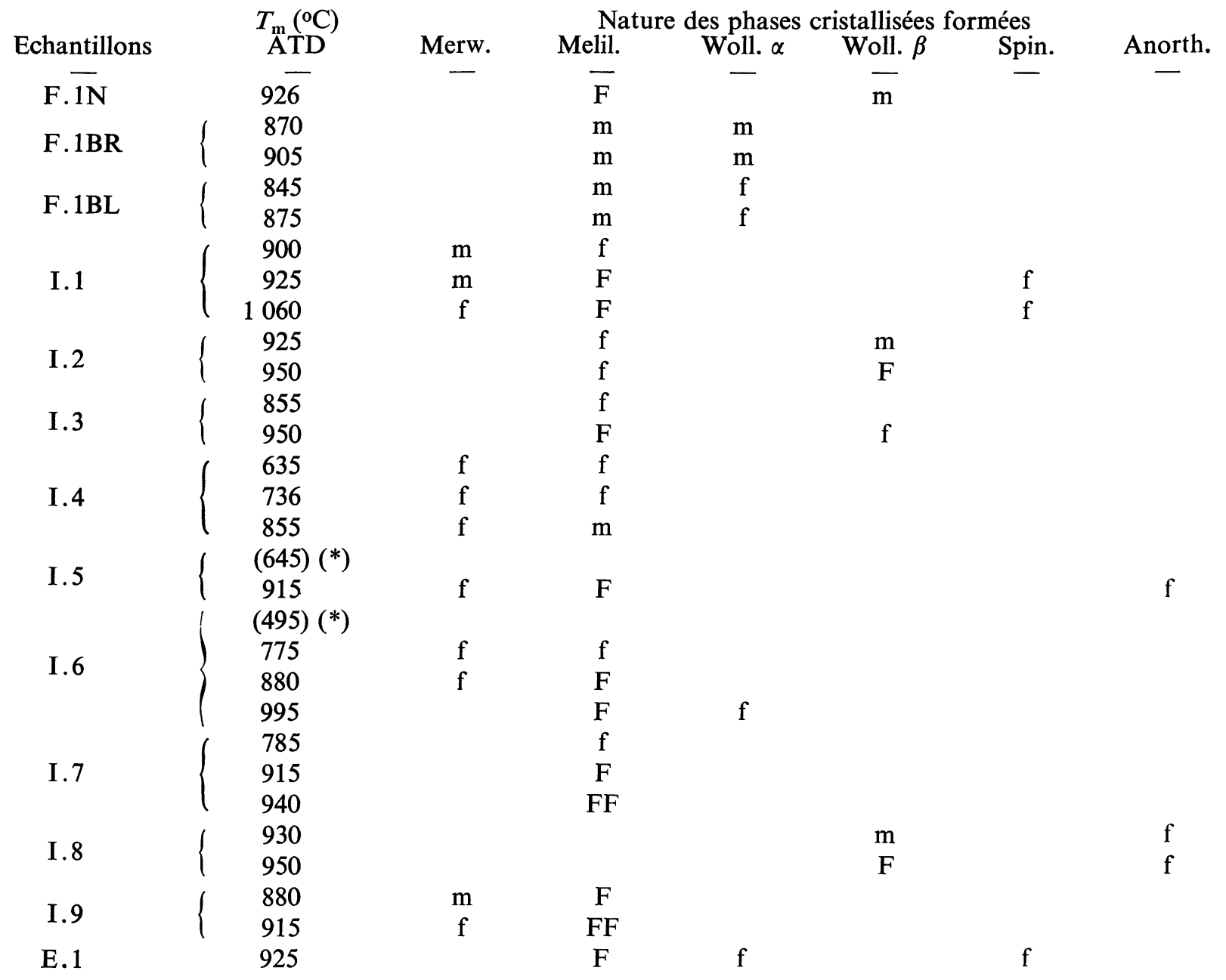

(*) Aucune phase cristalline ne se forme à ces deux températures.

dont la composition a été judicieusement choisie dans les systèmes $\mathrm{CaO}-\mathrm{Al}_{2} \mathrm{O}_{3}-\mathrm{SiO}_{2}, \mathrm{CaO}-\mathrm{MgO}-\mathrm{Al}_{2} \mathrm{O}_{3}-\mathrm{SiO}_{2}$, et $\mathrm{CaO}-\mathrm{Fe}_{2} \mathrm{O}_{3}$ (ou $\mathrm{FeO}$ ) $-\mathrm{Al}_{2} \mathrm{O}_{3}-\mathrm{SiO}_{2}$.

2.3 ETUDE DE LAITIERS SYNTHÉTIQUES DANS LES SYSTÈMES CaO- $\mathrm{Al}_{2} \mathrm{O}_{3}-\mathrm{SiO}_{2}, \mathrm{CaO}-\mathrm{MgO}-\mathrm{Al}_{2} \mathrm{O}_{3}-\mathrm{SiO}_{2}$, ET $\mathrm{CaO}-\mathrm{Fe}_{2} \mathrm{O}_{3}$ (oU $\mathrm{FeO}$ ). $\mathrm{Al}_{2} \mathrm{O}_{3}-\mathrm{SiO}_{2}$ [22-23]. - Des laitiers synthétiques dans les systèmes $\mathrm{CaO}-\mathrm{Al}_{2} \mathrm{O}_{3}-\mathrm{SiO}_{2}$ et $\mathrm{CaO}-\mathrm{MgO}-\mathrm{Al}_{2} \mathrm{O}_{3}-\mathrm{SiO}_{2}$ ont été préparés par fusion des mélanges d'oxydes ou de carbonates, puis trempe. Les compositions obtenues figurent dans le tableau VI et sur le diagramme de phase de la figure 14 (système exempt de $\mathrm{MgO}$ ).

Après trempe, ces matériaux ont été broyés, puis soumis à l'ATD. Connaissant l'allure des courbes ATD, des échantillons ont été ensuite traités thermiquement jusqu'à la température des sommets des pics exothermiques, puis refroidis à l'ambiante et analysés par diffraction $\mathrm{X}$. Certains ont été également chauffés à $1100^{\circ} \mathrm{C}$. L'examen des courbes ATD montre qu'à l'exception de l'échantillon 4, la dévitrification se manifeste par plusieurs pics exothermiques (Fig. 15).

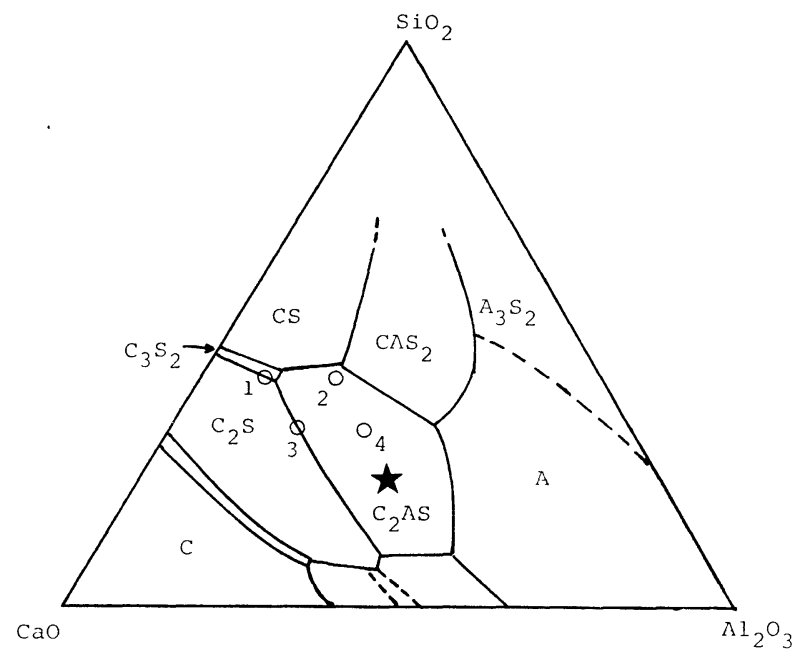

FIG. 14. - Position des laitiers synthétiques (numérotés de 1 à 4) dans le diagramme de phase du système $\mathrm{CaO}-\mathrm{Al}_{2} \mathrm{O}_{3}-\mathrm{SiO}_{2}$. $\mathrm{La}$ géhlénite est représentée par une étoile.

Les résultats de l'identification des phases cristallisées sont rassemblés dans le tableau VII. On n'a pas 
TABLEAU VI

\begin{tabular}{cc} 
Echantillons & $\mathrm{CaO}(\%)$ \\
\hline 1 & 50 \\
1A & 47,5 \\
1B & 45 \\
2 & 40 \\
$2 \mathrm{~A}$ & 38 \\
$2 \mathrm{~B}$ & 36 \\
3 & 50 \\
$3 \mathrm{~A}$ & 47,5 \\
$3 \mathrm{~B}$ & 45 \\
4 & 40 \\
4A & 38 \\
4B & 36
\end{tabular}

$\mathrm{Al}_{2} \mathrm{O}_{3}(\%)$
10
9,5
9,0
20
19
18
20
19
18
30
28,5
27

$\mathrm{SiO}_{2}(\%)$
40
38
36
40
38
36
30
28,5
27
30
28,5
27

\begin{tabular}{rc}
$\mathrm{MgO}(\%)$ & $\mathrm{CaO} / \mathrm{SiO}_{2}$ \\
\cline { 1 - 1 } 0 & 1,25 \\
5 & 1,25 \\
10 & 1,25 \\
0 & 1,00 \\
5 & 1,00 \\
10 & 1,00 \\
0 & 1,66 \\
5 & 1,66 \\
10 & 1,66 \\
0 & 1,33 \\
5 & 1,33 \\
10 & 1,33
\end{tabular}

TABLeAU VII

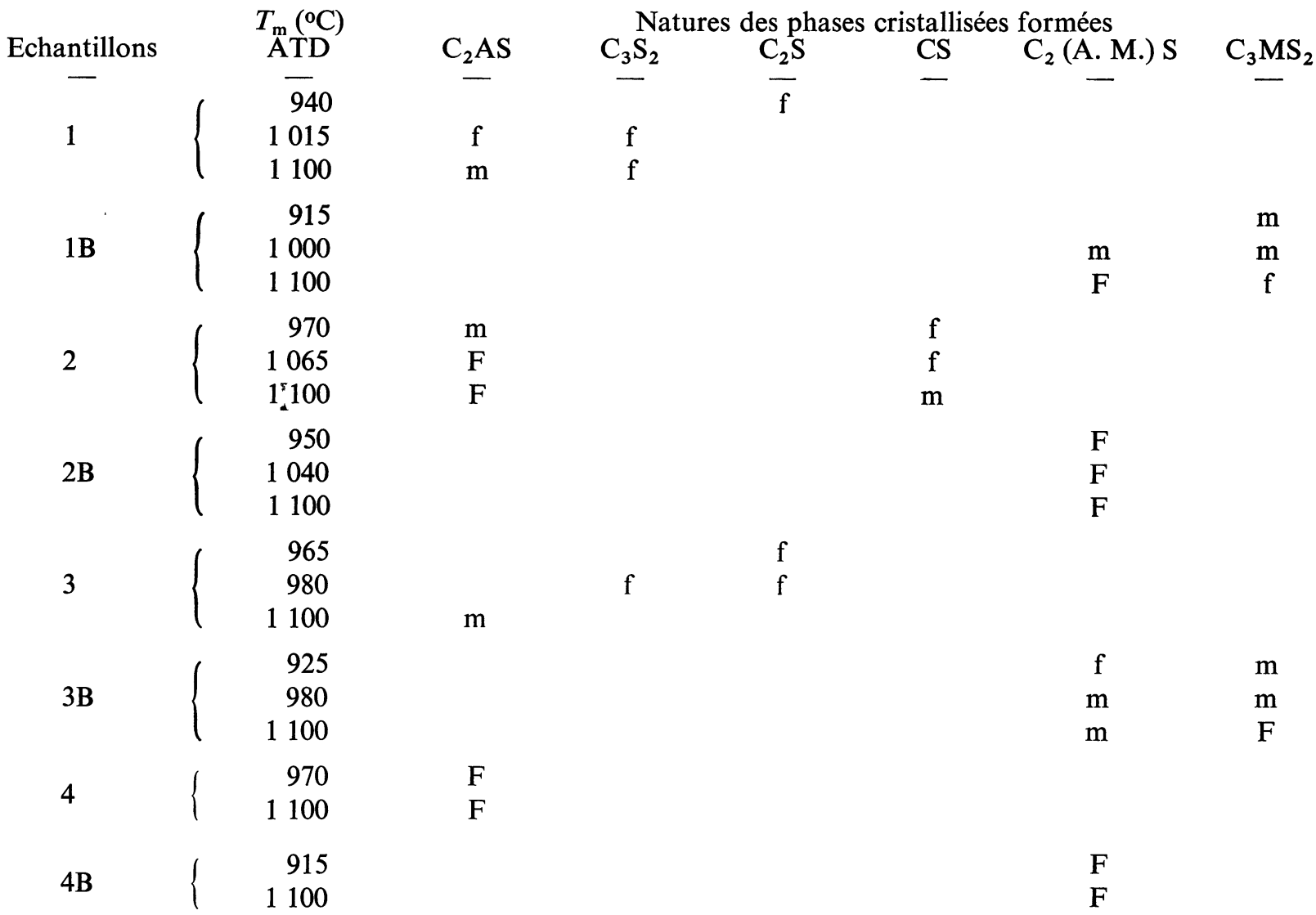

donné les résultats obtenus avec les laitiers à $5 \%$ de $\mathrm{MgO}$ car ils sont pratiquement identiques à ceux obtenus avec les échantillons à $10 \%$ de $\mathrm{MgO}$.

2.3.1 Echantillons exempts de MgO. Seul l'échantillon 4 conduit à la seule géhlénite. Les autres donnent de la géhlénite et d'autres phases appartenant aux domaines voisins de la géhlénite dans le diagramme de phase (Fig. 14). Ceci paraît normal pour l'échantillon 1 qui se trouve à la limite des domaines du silicate bicalcique $\mathrm{C}_{2} \mathrm{~S}$ et de la rankinite $\mathrm{C}_{3} \mathrm{~S}_{2}$. Le premirr cristallise à $940^{\circ} \mathrm{C}$, la rankinite à $1015^{\circ} \mathrm{C}$. Le silicate bicalcique réagit ensuite avec $\mathrm{CaO}$ et $\mathrm{Al}_{2} \mathrm{O}_{3}$ pour donner de la géhlénite $\mathrm{C}_{2} \mathrm{AS}$ selon le schéma :

$$
\text { Verre } \underset{(\mathrm{CaO})}{\longrightarrow} \mathrm{C}_{2} \mathrm{~S} \frac{\left(\mathrm{Al}_{2} \mathrm{O}_{3}\right)}{\stackrel{\left(\mathrm{Al}_{2} \mathrm{O}_{3}\right)}{\longrightarrow}} \mathrm{C}_{2} \mathrm{AS}
$$




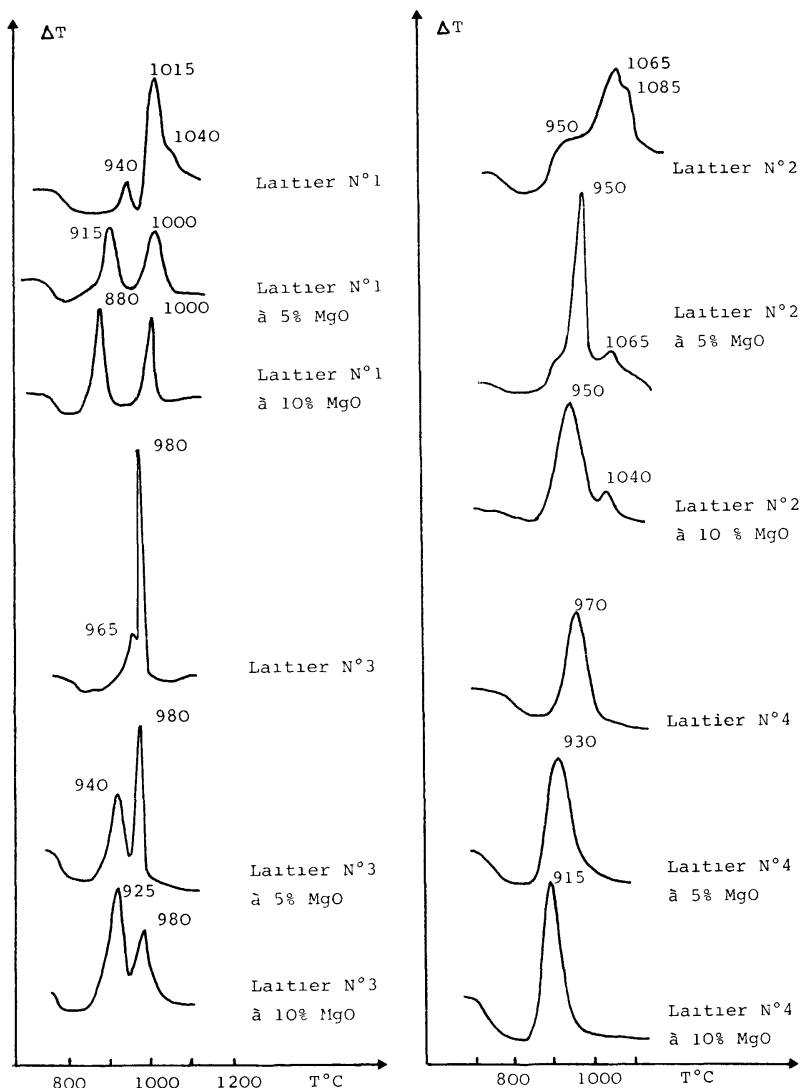

Fig. 15. - Courbes ATD des laitiers synthétiques dans les systèmes $\mathrm{CaO}-\mathrm{Al}_{2} \mathrm{O}_{3}-\mathrm{SiO}_{2}$ et $\mathrm{CaO}-\mathrm{MgO}-\mathrm{Al}_{2} \mathrm{O}_{3}-\mathrm{SiO}_{2}$.

Il n'est pas exclu que le laitier soit biphasique, une démixtion lors de la trempe ayant conduit à la formation de deux phases vitreuses $\mathrm{C}_{2} \mathrm{~S}$ et $\mathrm{C}_{3} \mathrm{~S}_{2}$.

La même hypothèse peut être faite pour l'échantillon 2 qui, bien que situé dans le domaine de la géhlénite, conduit à la cristallisation de cette phase et de wollastonite.

De même l'échantillon 3 conduit d'abord à la cristallisation du silicate bicalcique, puis de la rankinite. La géhlénite obtenue après traitement thermique à $1100^{\circ} \mathrm{C}$ résultant probablement de réactions à l'état solide.

Cette hypothèse de laitiers contenant plusieurs phases vitreuses, résultat d'une démixtion au refroidissement, mériterait confirmation par des examens de microscopie électronique après attaque de l'échantillon par des réactifs appropriés. Elle a déjà été vérifiée dans le cas de laitiers de haut-fourneau [24].

2.3.2 Echantillons contenant du magnésium. L'incorporation de $\mathrm{MgO}$ dans la composition des laitiers synthétiques modifie les courbes d'ATD (Fig. 15). La température des pics est abaissée et les phases qui cristallisent sont essentiellement la mélilite $C_{2}(A, M) S$ et la merwinite $\mathrm{C}_{2} \mathrm{MS}_{2}$. On retrouve l'influence du magnésium qui abaisse les températures de cristallisation (voir le paragraphe 2-1) et l'observation que dans le cas de laitiers vitreux contenant des teneurs déjà importantes en $\mathrm{MgO}$, la première phase qui cristallise est une phase contenant $\mathrm{MgO}$.

2.3.3 Laitiers synthétiques contenant $d u$ fer. Une série d'échantillons a également été préparée [23] avec des compositions identiques à celles présentées dans le tableau VI mais où $\mathrm{MgO}$ a été remplacé par $\mathrm{FeO}$ (addition d'oxalate ferreux au mélange lors de la synthèse). Les laitiers obtenus contiennent 0,75 ou $1,5 \%$ de $\mathrm{FeO}$, ce qui correspond aux compositions des laitiers industriels.

Les courbes ATD obtenues avec ces échantillons (Fig. 16, Fig. 17) montrent encore un abaissement de la

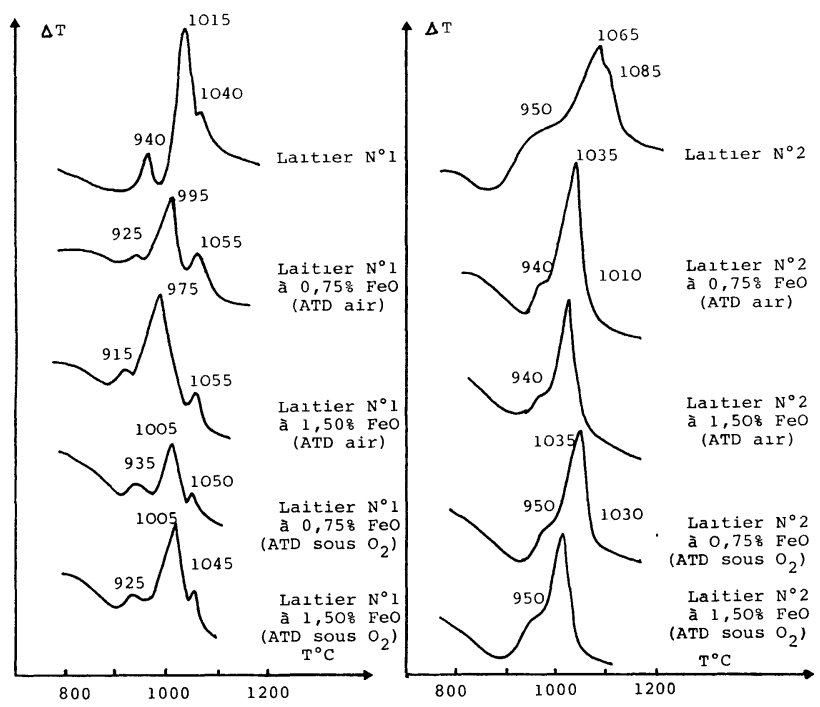

FIG. 16. - Courbes ATD des laitiers synthétiques contenant $\mathrm{du}$ fer dans le système $\mathrm{CaO}-\mathrm{Al}_{2} \mathrm{O}_{3}-\mathrm{FeO}-\mathrm{SiO}_{2}$.

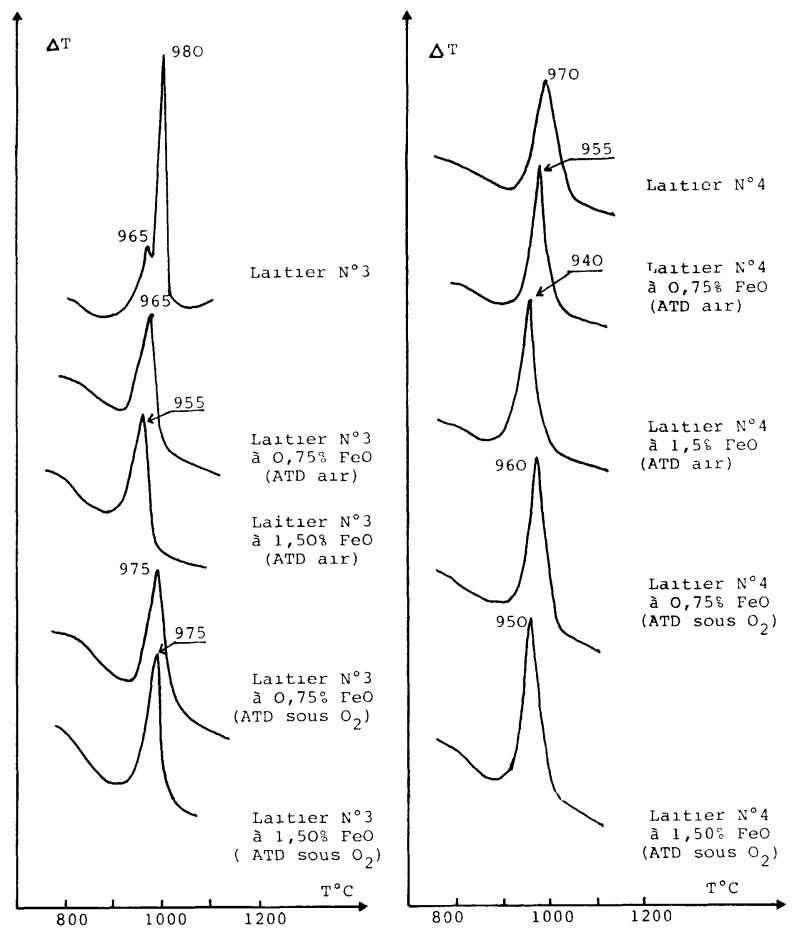

Fig. 17. - Courbes ATD des laitiers synthétiques contenant $\mathrm{du}$ fer dans le système $\mathrm{CaO}-\mathrm{Al}_{2} \mathrm{O}_{3}-\mathrm{FeO}-\mathrm{SiO}_{2}$. 
température des pics exothermiques de recristallisation lorsque l'échantillon contient du fer, cependant cet abaissement est moins marqué lorsque l'ATD est réalisée sous courant d'oxygène donc lorsque l'on produit l'oxydation du fer II en fer III.

La nature des phases qui cristallisent est modifiée par la présence de fer dans le verre : il se forme de la mélilite ferrique $\mathrm{C}_{2}(\mathrm{~F}, \mathrm{~A}) \mathrm{S}$ et de la wollastonite $\alpha$ ou $\beta$ selon que le chauffage est réalisé à l'air ou sous courant d'oxygène.

2. 4. Discussion. - Les résultats qui viennent d'être présentés montrent que l'ATD associée à la diffraction $\mathrm{X}$ permet de déterminer la température à laquelle se manifeste la recristallisation thermique des verres, de se faire une idée de la vitesse du phénomène par la forme du pic exothermique, et d'identifier les phases cristallisées qui se forment.

Toutes ces données sont nécessaires lorsque l'on veut élaborer des vitrocéramiques. On constate ainsi que la texture des vitrocéramiques obtenues à partir de verres de systèmes silicatés est très sensible à la façon dont se déroule la dévitrification : un pic d'ATD étroit correspond à une vitesse de cristallisation rapide, ce qui n'est pas toujours souhaitable car la vitrocéramique présente alors des microfissures [25], phénomène qui ne se manifeste pas lorsque le pic d'ATD est plus large. Le critère composition $d u$ verre semble encore plus important à prendre en considération : ainsi la présence de $\mathrm{MgO}$ dans le verre de départ dans le système $\mathrm{CaO}-\mathrm{Al}_{2} \mathrm{O}_{3}-\mathrm{SiO}_{2}$ provoque un dédoublement $\mathrm{du}$ ou des pics de dévitrification, donc modifie le processus de formation des phases cristallisées, ce qui conduit généralement à une vitrocéramique dont la texture est améliorée. De même la teneur en alumine dans le verre de départ doit être limitée sans quoi la vitrocéramique est plus fragile et présente des hétérogénéités. Le critère composition du verre est donc plus important que le critère «forme des pics $A T D$ ». On retrouve là une confirmation des limites de compositions indiquées par Loscei [26] dans le cas de l'utilisation des laitiers comme matière première dans l'élaboration des vitrocéramiques, à savoir :

\begin{tabular}{lr}
$\mathrm{SiO}_{2}$ & $28-40 \%$ \\
$\mathrm{Al}_{2} \mathrm{O}_{3}$ & $7-17 \%$ \\
$\mathrm{CaO}$ & $29-48 \%$ \\
$\mathrm{MgO}$ & $2-13 \%$ \\
$\mathrm{FeO}$ & $0,3-3 \%+$ traces de $\mathrm{BaO}, \mathrm{Na}_{2} \mathrm{O}, \mathrm{K}_{2} \mathrm{O}, \mathrm{TiO}_{2}$, \\
& \multicolumn{2}{|c}{$\mathrm{P}_{2} \mathrm{O}_{5}$, etc... }
\end{tabular}

Mais l'établissement de corrélations étroites entre la structure du verre de départ et la façon dont se manifeste la dévitrification est plus délicat. La courbe d'ATD permet de mettre en évidence une dévitrification en une ou plusieurs étapes selon que l'on obtient un ou plusieurs pics exothermiques, mais l'interprétation rigoureuse de ces phénomènes n'est pas toujours très facile. En effet on peut observer les différents cas suivants :
- apparition de plusieurs phases cristallisées, de nature différente, découlant d'un seul pic exothermique ;

- apparition d'une phase cristallisée donnée pouvant découler de la manifestation de deux pics exothermiques consécutifs.

- chaque pic d'ATD peut conduire à la formation d'une phase cristallisée donnée qui n'est pas forcément la même selon le pic considéré.

- dans le cas de l'addition d'agents de nucléation au verre de départ, dédoublement du pic exothermique avec cristallisation d'une phase métastable.

Relier ces phénomènes à la structure du verre n'est pas simple bien que l'hypothèse par exemple de la nature polyphasique du verre de départ (résultat d'une démixtion au refroidissement) ne soit pas à rejeter car déjà vérifiée dans de nombreux cas et par de nombreux auteurs [24-27].

Un point important qui ressort cependant des résultats présentés dans ce mémoire est que certains cations tels que $\mathrm{Mg}^{2+}$ et $\mathrm{Fe}^{3+}$ abaissent la température de cristallisation thermique, la première phase qui cristallise contenant ces cations. Ces phénomènes peuvent s'expliquer si on considère les valeurs de la force de champ ionique ou ionic field strength pour différents cations (Tableau VIII).

\section{TABLEAU VIII}

$\begin{array}{cccc}\text { Cations } & \text { Valence } Z & \begin{array}{c}\text { Rayon } \\ \text { ionique } r \\ (\text { en } \AA)\end{array} & \begin{array}{c}\text { Force } \\ \text { de champ } \\ \text { ionique } \\ \left(Z / r^{2}\right)\end{array} \\ \overline{\mathrm{Li}} & \overline{1} & \overline{-66} & \overline{2,78} \\ \mathrm{Na} & 1 & 0,95 & 1,11 \\ \mathrm{~K} & 1 & 1,33 & 0,57 \\ \mathrm{Mg} & 2 & 0,65 & 4,70 \\ \mathrm{Ca} & 2 & 0,99 & 2,04 \\ \mathrm{Sr} & 2 & 1,13 & 1,56 \\ \mathrm{Ba} & 2 & 1,35 & 1,10 \\ \mathrm{Fe} & 2 & 0,75 & 3,56 \\ \mathrm{Fe} & 3 & 0,63 & 7,60\end{array}$

Les ions à faible valeur de la force de champ ionique $\left(\mathrm{Na}^{+}, \mathrm{K}^{+}\right)$ne provoquent pas de séparation de phases car les forces électrostatiques qu'ils imposent sont trop faibles pour provoquer des distorsions importantes dans la structure du réseau silicaté du verre [2]. Par contre certains cations intersticiels à force de champ ionique élevée peuvent provoquer des perturbations dans le réseau silicaté du verre. Ainsi dans les systèmes $\mathrm{MgO}-\mathrm{SiO}_{2}$ et $\mathrm{CaO}-\mathrm{SiO}_{2}$ on peut s'attendre à des séparations de phases compte tenu des valeurs déjà élevées de $Z / r^{2}$ (4,7 pour $\mathrm{Mg}^{2+}$ et 2,04 pour $\mathrm{Ca}^{2+}$ ). Dans un système contenant à la fois les cations calcium et magnésium on peut penser que l'ion présentant la valeur la plus élevée de force de champ ionique (en l'occurence $\mathrm{Mg}^{2+}$ ) crée des distorsions plus importantes : il est donc normal de constater un abaissement 
de la température de dévitrification dans le système $\mathrm{CaO} \cdot \mathrm{MgO} \cdot \mathrm{Al}_{2} \mathrm{O}_{3} \cdot \mathrm{SiO}_{2}$, la phase qui cristallise la première étant celle qui contient le cation à plus forte valeur de $Z / r^{2}$. Ce phénomène que nous avons observé dans différents exemples mentionnés dans ce mémoire a été également mis en évidence par Sersale et Orsini dans le cas de l'étude de la dévitrification des verres de la solution solide géhlénite-merwinite [28].

Un phénomène analogue mais plus marqué se manifeste lorsque le verre contient du fer $\left(\mathrm{FeO}\right.$ ou $\left.\mathrm{Fe}_{2} \mathrm{O}_{3}\right)$, les valeurs de la force de champ ionique des cations $\mathrm{Fe}^{2+}$ et $\mathrm{Fe}^{3+}$ étant bien supérieures à celle de $\mathrm{Ca}^{2+}$.

Par ailleurs l'augmentation de la teneur en alumine dans le verre se traduit par une élévation de la température de dévitrification. L'aluminium stabilise donc le verre (contrairement au magnésium ou au fer), son rôle est cependant différent de celui d'un cation modificateur de réseau. Des teneurs assez élevées en $\mathrm{Al}_{2} \mathrm{O}_{3}$ dans les verres des systèmes $\mathrm{CaO}-\mathrm{Al}_{2} \mathrm{O}_{3}-\mathrm{SiO}_{2}$ ou $\mathrm{CaO}-\mathrm{MgO}-\mathrm{Al}_{2} \mathrm{O}_{3}-\mathrm{SiO}_{2}$ inhibent même de façon très nette la cristallisation du verre lors du refroidissement lent de celui-ci [29].

La façon et la température à laquelle se manifeste la recristallisation permet donc de se faire une idée de la stabilité donc de la structure du verre de départ.

Une approche cinétique du phénomène de dévitrification pourrait apporter des informations plus précises : en effet la connaissance du mécanisme et de la valeur du travail de formation des germes, pour un verre donné, permettrait de proposer un modèle de structure pour ce verre.

Cependant des études cinétiques valables ne peuvent, à notre avis, être obtenues à partir de simples enregistrements d'ATD. La calorimétrie serait certainement mieux adaptée à ce problème. Il semble que c'est dans cette voie qu'il faudrait s'engager maintenant.
3. Conclusion. - L'élaboration de vitrocéramiques à partir de matériaux vitreux (silicates par exemple) nécessite une connaissance préalable du comportement thermique de la matière première. La caractérisation de cette dernière peut être effectuée par des techniques simples telles que l'analyse thermique différentielle (ATD) et la diffraction des rayons $\mathrm{X}$. On constate que la température de dévitrification est étroitement liée à la composition du verre de départ et de sa structure. Cette dévitrification peut se manifester par un ou plusieurs pics exothermiques en ATD. L'allure de ces pics renseigne sur la vitesse de cristallisation, la déflexion endothermique qui les précède sur la nucléation.

La température à laquelle se manifeste la cristallisation est généralement abaissée lorsque le verre contient plusieurs cations (modificateurs de réseau) qui diffèrent par leur force de champ ionique. Le cation qui présente la valeur la plus élevée de force de champ ionique se retrouve dans la première phase qui cristallise (cas de $\mathrm{Mg}^{2+}$ et de $\mathrm{Fe}^{3+}$ dans les verres des systèmes silice-chaux-alumine).

Lorsqu'une même phase résulte d'une cristallisation à deux ou plusieurs pics exothermiques, ou que ces pics conduisent à la cristallisation de phases différentes, on peut penser que le verre de départ est polyphasique (existence de plusieurs phases vitreuses), donc qu'il y a eu démixtion au refroidissement lors de la préparation de ce verre. Une telle hypothèse nécessiterait cependant une vérification par les techniques de microscopie électronique, tout du moins dans le cas des exemples présentés ici.

Une interprétation cinétique des courbes d'analyse thermique devrait apporter des informations complémentaires quant à la structure du verre mais il existe encore peu de données cohérentes à ce sujet.

\section{Bibliographie}

[1] Scholze, H., Le Verre, (Ed. Institut du Verre, Paris), 1968.

[2] McMillan, P. W., Glass-Ceramics (Acad. Press, London and New-York) 1964

[3] WilbuRN, F. W. et Dawson, J. B., Glass dans Differential Thermal Analysis, Ed. R. C. Mackenzie (Acad. Press, London and New-York) 2 (1972) 229.

[4] Thakur, R. L., Takizawa, K., Sakaimo, T., Moriya, T., Bull. Cent. Glass Ceram. Res. Inst., Calcutta, 11 (1964) 1.

[5] Mercier, M., Silic. Ind. (Belg.) 32 (1975) 159.

[6] Amato, I. et Negro, A., Ceramurgia (Ital.) 1 (1971) 16

[7] Negro, A., Murat, M., SASSI, F., Ceramurgia Int., 2 (1976) 131

[8] Negro, A. et Amato, I., Ceramurgia (Ital.), 3 (1973) 77

[9] Negro, A. et Amato, I., Ceramurgia (Ital.) 3 (1973) 133.

[10] Amato, I. et Negro, A., Ceramurgia (Ital.) 2 (1972), 190.

[11] Amato, I. et Negro, A., Ceramurgia (Ital.) 4 (1974) 237.

[12] Sosulja, P. W. et Wachtler, H. J., J. Thermal Anal. 7 (1975) 643.

[13] Negro, A., Journées d'Information de l'I. N. S. A. de Lyon sur les Déchets et Sous-produits Industriels : Elimination, Recyclage, Valorisation. Lyon, nov. 1975. Ed. I. N. S. A., (1975), XVII (12 pages)

[14] LagneauX, H., Ibid., XVI (7 pages).

[15] Kondo, R., VIth. Intern. Symp. Chem. Cem., Washington (1960), 973.
[16] Bonetti, G., Sprechsaal Keram.-Glas-Email (1963), 5.

[17] SCHRÄMLI, W., Zem.-Kalk-Gips 140 (1963) 4.

[18] Krüger, J. E. et Smith, M. S., Cem.-Lime Manuf. 77 (1969) 42.

[19] Terrier, P., CILAM Inform., (1973), no 8, 1.

[20] Negro, A. et Murat, M., Thermal Analysis, Proceed. of I. C. T. A. IV, Budapest, 3 (1975) 635.

[21] Murat, M. et Negro, A., Revue Phys. Appl. 9 (1974) 479 ; Mat. and Struct. (RILEM Bull.) (1974) 253.

[22] Negro, A. et BAChIORrini, A., Zem.-Kalk-Gips 9 (1973) 448.

[23] SASSI, F. et BAChiorrini, A., Colloque Intern. sur les I.aitiers, les Scories, et les Déchets. Mons (Belg.), 1012 sept. 1975 .

[24] SCHRöDER, F., Tonind. Ztg., (1961) 39; Vth. Intern. Symp. Chem. Cem., Tokyo, IV (1968) 149.

[25] Negro, A. et Bachiorrini, A., Ceramurgia (Ital.) 4 (1974) 11.

[26] LocseI, B. P., Interceram. 2 (1966) 133.

[27] Cahn, J. W. et Charles, R. J., Phys. and Chem. of Glasses 6 (1965) 181-91.

[28] Sersale, R. et Orsini, G., Silic. Industr. (Belg.) 34 (1969) 83.

[29] Amato, I., Negro, A. et BaChiorrini, A., Rev. Int. Hautes Temp. et Réfract. 12 (1975) 241. 